\title{
Experimental constraints on the astrophysical interpretation of the cosmic ray Galactic-extragalactic transition region
}

\author{
C. De Donato ${ }^{a}, *$, G. A. Medina-Tanco ${ }^{\mathrm{b}, * *}$ \\ ${ }^{a}$ Dipartimento di Fisica, Università degli Studi di Milano and INFN, Milano, Italy \\ ${ }^{\mathrm{b}}$ Dep. Altas Energias, Inst. de Ciencias Nucleares, Universidad Nacional \\ Autonoma de México, México DF, CP 04510
}

\begin{abstract}
The energy region spanning from $\sim 10^{17}$ to $\lesssim 10^{19} \mathrm{eV}$ is critical for understanding both, the Galactic and the extragalactic cosmic ray fluxes. This is the region where the propagation regime of nuclei inside the Galactic magnetic environment changes from diffusive to ballistic, as well as the region where, very likely, the most powerful Galactic accelerators reach their maximum output energies. In this work, a diffusion Galactic model is used to analyze the end of the Galactic cosmic ray spectrum and its mixing with the extragalactic cosmic ray flux. In particular, we study the conditions that must be met, from the spectral and composition points of view, by the Galactic and the extragalactic fluxes in order to reproduce simultaneously the total spectrum and elongation rate measured over the transition region by HiRes and Auger. Our analysis favors a mixed extragalactic spectrum in combination with a Galactic spectrum enhanced by additional high energy components, i.e., extending beyond the maximum energies expected from regular supernova remnants. The two additional components have mixed composition, with the lowest energy one heavier than the highest energy one. The potential impact on the astrophysical analysis of the assumed hadronic interaction model is also assessed in detail.
\end{abstract}

Key words: cosmic rays, Galactic deconfinement, composition and spectrum $P A C S$ :

\footnotetext{
* Corresponding author. Dipartimento di Fisica, Università degli Studi di Milano, via Celoria 16, Milano, CAP 20133, Italy. Telephone number: ++39 0250317710

${ }^{* *}$ Corresponding author. Instituto de Ciencias Nucleares, UNAM, Apartado Postal 70-543, Ciudad Universitaria, Mexico D.F. 04510, Mexico. Telephone number: $++52-55-5622-4690$ (ext.: 333), fax number: ++52-55-5622-4693

Email addresses: cinzia.dedonato@mi.infn.it (C. De Donato), gmtanco@nucleares. unam.mx (G. A. Medina-Tanco).
} 


\section{Introduction}

The cosmic ray (CR) energy spectrum extends for many orders of magnitude with a power law index $\approx 2.7$. Along this range of energies, the three spectral features are known: the first knee at $E \approx 3 P e V$, the second knee at $E \approx 0.5 \mathrm{EeV}$ and the ankle, a dip extending from the second knee to beyond $10 \mathrm{EeV}$. The nature of the second knee and of the ankle is still uncertain [1]; a possible interpretation of the two features is the transition between the Galactic and extragalactic components. At energies between $10^{17}-10^{18} \mathrm{eV}$ the Galactic supernova remnants (SNR) are expected to become inefficient as accelerators. This fact, combined with magnetic deconfinement, should mark the end of the Galactic component of cosmic rays, although the picture could be confused by the existence of additional Galactic accelerators at higher energy. On the other hand, at energies above the second knee, extragalactic particles are able to travel from the nearest extragalactic sources in less than a Hubble time. Consequently, the spectrum may present above $10^{17.5} \mathrm{eV}$ a growing extragalactic component that becomes dominant above $10^{19} \mathrm{eV}$. The region between the second knee and the ankle could be the transition region between the Galactic and extragalactic components.

In this work we analyze the transition region comparing the diffusive Galactic spectrum from SNRs with two different models of extragalactic spectrum, one in which only protons [2] are injected at the sources and another in which a mixed composition containing heavy nuclei 3] is injected. To discriminate between the possible astrophysical scenarios we analyze the composition parameter $X_{\max }$ inferred from the different extragalactic (EG) models using the hadronic interaction models used in literature. A comparison with the experimental data is done with special attention to Auger and HiRes data.

\section{Galactic and extragalactic cosmic rays: transition models}

The nature of the knee, consisting of a steepening of the spectrum from a power low index $\gamma_{g}=2.7$ to $\gamma_{g}=3.1$, is well explained by the rigiditymodels (rigidity-acceleration model and rigidity-confinement model), in which the maximum energy achievable by nuclei is rigidity dependent. In these models, the knees of the spectrum of nuclei of charge $\mathrm{Z}$ are related to the proton knee energy $E_{k n}^{Z}=Z E_{k n}^{p}$, where $E_{k n}^{p}=2.5 \times 10^{15} \mathrm{eV}$. Beyond the highest energy knee $E_{k n}^{F e}=6.5 \times 10^{16} \mathrm{eV}$ the total Galactic flux, dominated by the Iron component, must be steeper. While this spectral feature seems to be confirmed by KASCADE data [4], the nature of the second knee and of the ankle are still

uncertain [1]. Although the region between the second knee and the ankle is 
naturally considered as the transition between the Galactic and extragalactic component, the way the transition takes place is very model dependent. The main models describing the transition are the ankle model, the dip model and the mixed composition model.

In the ankle model the transition between the Galactic cosmic rays (GCR) and extragalactic cosmic rays (EGCR) occurs at the ankle $E_{a} \approx 1 \times 10^{19} \mathrm{eV}$ [5, 6, 7,8,9,10,11,12. The dip in the CR spectrum is explained as the intersection of a flat extragalactic component with the steep Galactic component. An advantage of this model is the extragalactic flat generation spectrum $E^{-2}$ which provides reasonable luminosities of the sources. On the other hand, the ankle model doesn't work well in the framework of the rigidity-model. If the transition from Galactic to extragalactic CRs occurs at the ankle, other additional Galactic mechanisms are required to accelerate particles at energies beyond $E_{k n}^{F e}=6.5 \times 10^{16} \mathrm{eV}$.

A different explication of the dip and a lower transition energy comes from the dip model [132]. In this model the dip is caused by $e^{+} e^{-}$pair production by the extragalactic protons with the CMB photons. The transition energy is determined by the energy at which adiabatic energy losses are equal to pair production energy losses. In this case the transition between the Galactic component and the extragalactic component takes place at lower energies, around the second knee, in agreement with the rigidity-model. The calculated position and shape of the dip, confirmed by Akeno-AGASA [14], HiRes [15], Yakutsk [16,17] and Fly's Eye [18] experimental data 1, is "universal" as it doesn't depend on the type of propagation, on the source density and separation (for separation distance $d<100 \mathrm{Mpc}$ ) and on the evolutionary model of the sources. The only factor that affect the dip is a heavy component of the EG spectrum [20]21]. The shape and position of the dip is in agreement with observations for an EG pure proton composition with a maximum contamination of Helium nuclei of the order of $10 \%$.

On the other hand, as the dip model requires a generation spectrum $E^{-2.7}$, a solution is needed to prevent the too high emissivity needed at lower energies $[13,22,2,23]$.

An alternative and intermediate model is the mixed composition model [24,25,3]. In this model the EG cosmic ray composition is assumed to be mixed, in analogy with the Galactic component. As in the ankle model, the intersection of the Galactic and extragalactic component gives origin to the the dip structure but with the advantage of a lower transition energy (around $E \approx 3 \times 10^{18} \mathrm{eV}$ ), which softens the requirement of additional acceleration mechanism. The calculated spectrum, fitted with the observed data, presents a spectral index

1 Auger data don't contradict the high energy part of dip but an extension of Auger to lower energies it's needed to confirm the feature of the dip [19]. 
$\gamma \approx 2.1-2.3$, which also provides a reasonable luminosity.

The three model can be experimentally distinguished through measurements of the spectrum and of anisotropies, although the most discriminant feature is the chemical composition. While in the ankle model the Galactic heavy component dominates up to the ankle energy, in the dip model the transition is completed at energy around $1 \times 10^{18} \mathrm{eV}$ where the composition is proton dominated. The composition at this energy (Iron/proton) is a discriminant between the two models. In the case of the mixed model, the transition occurs at $E \approx$ $3 \times 10^{18} \mathrm{eV}$ and the chemical composition in the dip region is mixed. This model predicts a slower decrease of the Fe component and a slower increase of the proton fraction in the transition energy range.

\section{Galactic-extragalactic spectrum}

The current paradigm for Galactic cosmic ray acceleration is the Fermi acceleration mechanism by shock waves of SuperNova Remnants (SNRs) [26].

In this section we calculate the Galactic diffusive spectrum from SNRs using the numerical diffusive propagation code GALPROP [27,28].

The calculated Galactic spectrum from SNRs is then combined with two different models of EG spectrum, one in which only protons [2] are injected at the sources and another in which a mixed composition containing heavy nuclei [3] is injected instead.

We analyze the transition region between Galactic and extragalactic components in the two different EG scenarios and compare the combined total spectrum with the available experimental data.

\subsection{Diffusion Galactic model}

We use the numerical diffusive propagation code GALPROP [27,28] to reproduce the Galactic spectrum from SNRs.

The diffusive model is axisymmetric. The propagation region is, in cylindrical coordinates, bounded by $R=R_{h}=30 k p c$ and $z=z_{h}=4 k p c$, beyond which free escape is assumed. The diffusion equation is:

$$
\frac{\partial \psi}{\partial t}=q(\vec{r}, p)+\vec{\nabla} \times\left(D_{x x} \vec{\nabla} \psi\right)-\frac{\partial}{\partial p}(\dot{p} \psi)-\frac{1}{\tau_{f}} \psi-\frac{1}{\tau_{r}} \psi
$$

where $\psi(\vec{r}, p, t)$ is the density per unit of total particle momentum, $q(\vec{r}, p)$ is the source term, $D_{x x}$ is the spatial diffusion coefficient, $\dot{p}=d p / d t$ is the 
momentum loss rate and $\tau_{f}$ and $\tau_{r}$ are the time scale of fragmentation and the time scale of radioactive decay respectively.

The GALPROP code solves the diffusion equation for all cosmic-ray species starting from the heaviest nucleus and then proceeding to lighter nuclei using the computed secondary source functions. The numerical solution of the transport equation is based on a Crank-Nicholson [29,27] implicit second-order scheme.

The diffusion coefficient is taken as $\beta D_{0}\left(\rho / \rho_{D}\right)^{\delta}$, where $\rho$ is the particle rigidity, $D_{0}$ is the diffusion coefficient at a reference rigidity $\rho_{D}$ and $\delta=0.6$. The diffusion coefficient can be inferred from the abundances of light nuclei, produced mainly through spallation of heavy elements, as Li, Be and B, which give an estimation of the time of residence of CRs in the galaxy of $\approx 1.5 \times 10^{7} \mathrm{yr}$ [30]. We use $D_{0}=5.75 \times 10^{28} \mathrm{~cm}^{2} \mathrm{~s}^{-1}$ at the reference rigidity $\rho_{D}=4 \mathrm{GV}$.

The assumption of a diffusion coefficient with an energy dependence $E^{-0.6}$ is not universally accepted [31]. In fact, the turbulence in the interstellar medium seems to follow closely a Kolmogorov spectrum, which should lead to an energy dependence of $E^{1 / 3}$ for the diffusion coefficient. In this scenario, the difference between primary and secondary cosmic ray energy spectra could be explained by the trapping of primary cosmic rays in high density regions with Kolmogorov turbulence near their acceleration sites, where secondary CR would be mainly produced, and the diffusion of the latter inside this region and the interstellar medium. However, since at high energy both models produce similar spectra and composition profiles, we think our analysis is to a large extent independent of these assumptions.

The assumed distribution of cosmic rays sources is the one reproducing the cosmic-ray distribution determined by the analysis of EGRET gamma-ray data, which has the same parameterization of the R-dependence as that used for SNRs [27]:

$$
f(R, z)=f(R) \exp \left(-|z| / z_{\text {scale }}\right)
$$


where $z_{\text {scale }}=0.2 \mathrm{kpc}$ and:

$$
f(R)= \begin{cases}19.3 & R \leq 4 k p c \\ 21.9 & 4 k p c<R \leq 8 k p c \\ 15.8 & 8 k p c<R \leq 10 k p c \\ 18.3 & 10 k p c<R \leq 12 k p c \\ 13.3 & 12 k p c<R \leq 15 k p c \\ 7.4 & R>15 k p c .\end{cases}
$$

This choice is due to the fact that the SNR distribution [32] produces CR distribution distinctly different from the measurements. A solution to the apparent discrepancy between the radial gradient in the diffuse Galactic gamma-ray emissivity and the distribution of SNRs was proposed by Strong et al. [33.

According to shock acceleration models [34]35], the injection spectrum is a power law function in rigidity with a break at rigidity $\rho_{0}$, beyond which it falls exponentially with a rigidity scale $\rho_{c}$ :

$$
I(\rho)= \begin{cases}\left(\frac{\rho}{\rho_{0}}\right)^{-\alpha} & \rho<\rho_{0}, \\ \left.\exp \left[-\left(\frac{\rho}{\rho_{0}}-1\right) / \frac{\rho_{c}}{\rho_{0}}\right)\right] & \rho>\rho_{0},\end{cases}
$$

where $\rho_{0}=1.8 P V, \rho_{c}=1.26 P V$ and $\alpha=2.05$, which is the case of strong shock waves $(M>>1)[36,37$.

Stable nuclei with $Z \leq 26$ are injected, with energy independent isotopic abundances derived from low energy CR measurements [28,,38,39,40,41,42, 43].

The interstellar hydrogen distribution, molecular, atomic and ionized (H2, HI, $\mathrm{HII}$ ), is derived from radio $\mathrm{HI}$ and CO surveys in 9 Galactocentric rings and information on the ionized component [44]. The distribution of molecular hydrogen is derived indirectly from $\mathrm{CO}$ radio-emission and the assumption that the conversion factor $\mathrm{H} 2 / \mathrm{CO}$ is the same for the whole Galaxy [45]. The atomic hydrogen (HI) distribution is taken from [46], with a z-dependence calculated using two approximation at different Galactocentric distance $\mathrm{R}$ [47[48]. The ionized component HII is calculated using a cylindrically symmetrical model [49].

The Interstellar Radiation Field (ISRF) is calculated using emissivities based on stellar populations and dust emission [50,51].

We consider a Galactic turbulent magnetic field with a mean value of the 
component perpendicular to the CR propagation given by

$$
B=B_{0} \exp \left(-\frac{R-R_{0}}{R_{\text {scale }}}\right) \exp \left(-\frac{|z|}{z_{\text {scale }}}\right),
$$

where $B_{0}=6 \mu G, R_{\text {scale }}=10 \mathrm{kpc}, z_{\text {scale }}=2 \mathrm{kpc}$ and $R_{0}=8.5 \mathrm{kpc}$ the Sun Galactocentric distance.

\subsection{Diffusive Galactic spectrum}

The calculated diffusive Galactic spectrum is shown in Fig.1 superimposed to several experimental data results from the air shower experiments (HiRes [52, HiResMIA [53, Fly's Eye [18, KASCADE [4, Yakutsk [16], Akeno [54,55], AGASA [14, Haverah Park [56,57], Auger [19,58], BLANCA [59], Tibet 60,61, Mt. Norikura 62]) as well as from direct measurements (SOKOL [63], JACEE [64,65], Grigorov [66], Proton Satellite [67], Runjob [68]).

It can be seen that there is considerable dispersion over the whole energy interval, which highlights the inherent difficulty in CR spectral measurements over so many decades in energy. Furthermore, it can be seen that, below the first knee the dispersion is mainly limited to flux normalization, while the spectral indexes seem to be fairly consistent for different experiments. The situation worsens at higher energies until large discrepancies are apparent above $10^{17}$ $\mathrm{eV}$. However, even at these high energies, a careful renormalization in energy [69] seems to be able to reconcile the several experimental data sets, giving a rather clear and consistent picture for the ankle. Unfortunately, normalization flux differences among spectra still remain between major experiments after the previous energy scale shifting, and they represent a concern to be experimentally addressed.

The diffusive Galactic spectrum $\Phi_{G}$ is normalized to match KASCADE data at $\sim 3 \times 10^{6} \mathrm{GeV}$ [4. At this energy the differential flux value of the all particle energy spectrum and its uncertainty are the same for QGSJet 01 and Sibyll 2.1 based analysis of the KASCADE experiment. While, with this renormalization, our spectrum agrees with data of various experiments at lower energies (JACEE, SOKOL, Tibet, KASCADE, Haverah Park and Akeno), beyond the knee the diffusive spectrum presents a strong deficit of flux (Fig,1).

Since at $E>10^{7} \mathrm{GeV}$ the composition is dominated by intermediate $(Z$ : $6-12$, i.e the CNO group) and heavier $(Z: 19-26)$ nuclei, we renormalize these components $\left(\Phi_{G}^{C N O}, \Phi_{G}^{H}\right)$ by a factor of 2 , which produces a good agreement with the experimental data (see Fig,2).

The renormalization of the CNO group and of the heavy nuclei group by a fac- 


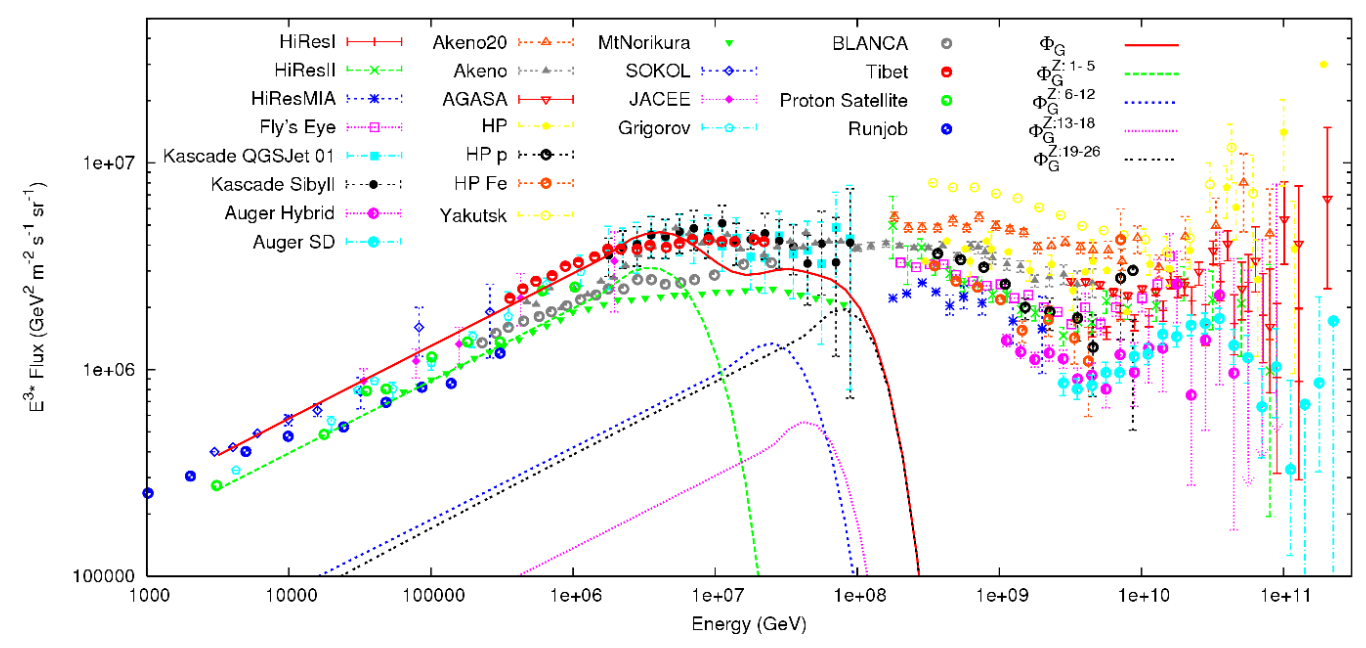

Fig. 1. Diffusive Galactic spectrum, $\Phi_{G}$. The different Z-grouped nuclei components are shown.

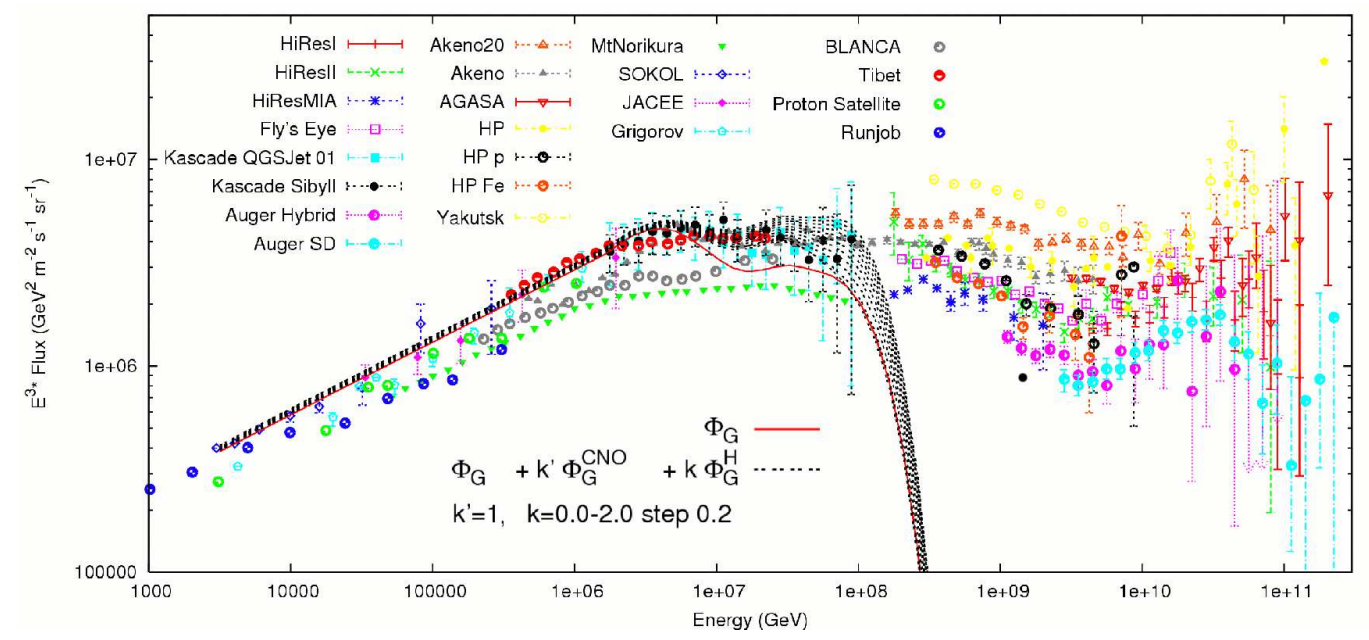

Fig. 2. Diffusive Galactic spectrum, $\Phi_{G}$, with renormalization of the CNO group $(Z: 6-12), \Phi_{G}^{C N O}$, and of the heavy component $(Z: 19-26), \Phi_{G}^{H}$. Agreement with experimental data is obtained for $k^{\prime} \approx 1$ and $k \approx 0.8-1.2$.

tor of 2 is acceptable since it corresponds to a renormalization of the injected abundances into the acceleration mechanism. The Galactic CR CNO nuclei are over abundant with respect to Iron by several orders of magnitude. Therefore, the Iron contribution to CNO CR flux resulting from spallation is small. Consequently, to a good approximation, the observed Galactic abundances of Iron and the CNO group at the Solar circle can be varied independently by modifying their relative abundances at injection. This amount of relative renormalization is well inside the present uncertainties regarding the detailed workings of the injection mechanism.

The renormalized diffusive Galactic spectrum reproduces well the data up to $E \approx 10^{8} \mathrm{GeV}$, beyond which the spectrum falls steeply because of the end of the SNR acceleration efficiency. 


\subsection{Extragalactic spectrum}

In order to study how the transition between the Galactic and extragalactic components takes place, we compare the Galactic spectrum originated in SNRs with two different possible scenarios for the extragalactic component.

In the first model [2], a pure proton extragalactic spectrum, accelerated by a homogeneous distribution of cosmic sources, is considered. Local overdensities/deficits of UHECR sources affect the shape of the GZK modulation, but do not affect the low energy region where the matching with the Galactic spectrum occurs. Within this model, we consider different cases of local overdensity/deficit of sources:

(1) universal spectrum: $n / n_{0}=1$;

(2) overdensity of sources: $n / n_{0}=2, R=30 \mathrm{Mpc}$;

(3) overdensity of sources: $n / n_{0}=3, R=30 M p c$;

(4) deficit of sources: $n / n_{0}=0, R=10 \mathrm{Mpc}$;

(5) deficit of sources: $n / n_{0}=0, R=30 \mathrm{Mpc}$;

where $n_{0}$ is the mean extragalactic source density and $n$ is the local overdensity/deficit in regions of size $R$.

In the second model [3], the extragalactic spectrum is calculated for a mixed composition at injection typical of low energy cosmic rays. The spectral in$\operatorname{dex} \beta$ is determined fitting the high energy CR data. Three different source evolution models in red shift are considered:

a) strong evolution model: the injection rate is proportional to $(1+z)^{4}$ for $z<1$ and constant for $1<z<6$, followed by a sharp cut-off, $\beta=2.1$;

b) SFR model: the EGCR injection power is proportional to the star formation rate which correspond to a redshift evolution $(1+z)^{3}$ for $z<1.3$ and a constant injection rate for $1.3<z<6$ (with a sharp cut-off at $z=6$ ), $\beta=2.2$

c) uniform source distribution model: no evolution, $\beta=2.3$.

In both cases, the various parameters of the models are tuned to fit the available CR data at UHE and are, in that highest energy regime, experimentally indistinguishable at present. The spectra of both the models, used in the next sections, are renormalized to HiRes data. 


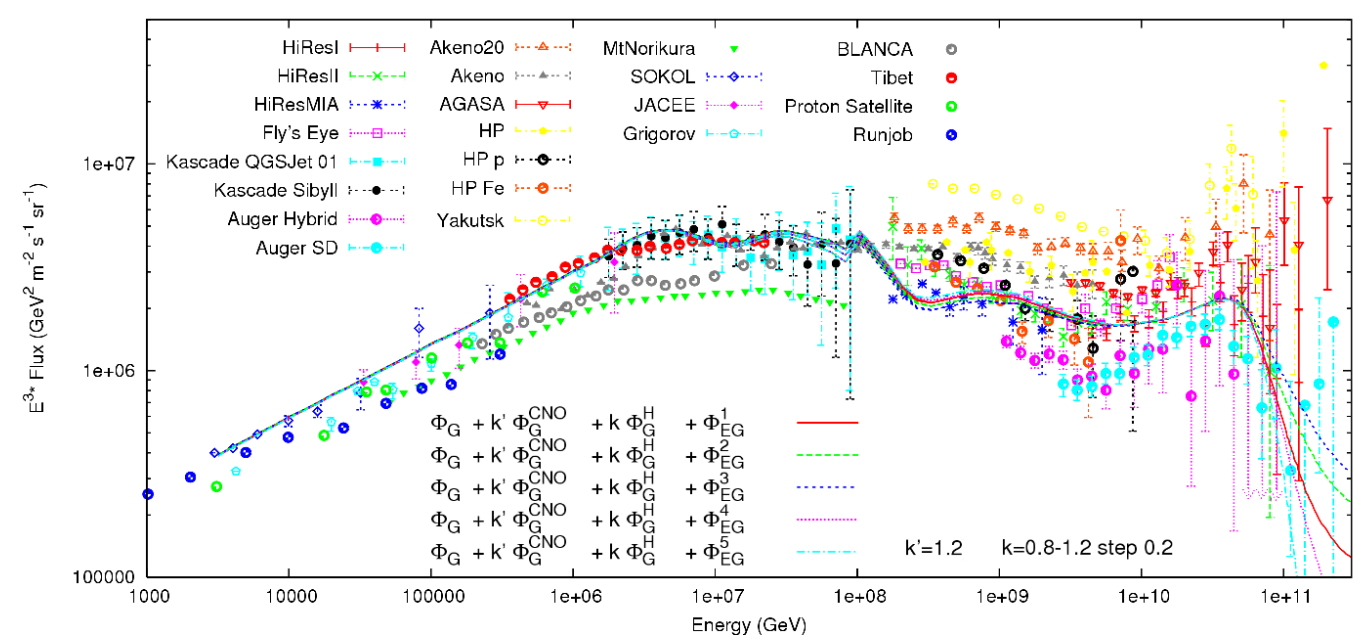

Fig. 3. Galactic and extragalactic spectrum matching for the proton models for an EG lower energy limit of $10^{8} \mathrm{GeV}$. The sum of the renormalized diffusive Galactic spectrum and of the extragalactic spectrum $\left(\Phi_{E G}\right)$ for different cases of local overdensity/deficit of sources (\$2.3) is shown for different renormalizations of the heavy component $\left(\Phi_{G}^{H}\right)$. The CNO group $\left(\Phi_{G}^{C N O}\right)$ of the diffusive Galactic spectrum $\left(\Phi_{G}\right)$ has been renormalized by a factor 2.2 .

\subsection{Combined spectrum: matching Galactic and extragalactic components}

In order to study how the transition between the Galactic and extragalactic components takes place, we subtract the combined theoretical (Galactic $\Phi_{G}$ plus extragalactic $\left.\Phi_{E G}\right)$ spectrum from the available data. Two different approaches are used.

First, we try to match the experimental data by varying the normalization of the heavy Galactic component $\Phi_{G}^{H}$, while keeping constant the previous renormalization of the CNO group $\Phi_{G}^{C N O}(\$ 2.2)$. The best reproduced spectrum for the two extragalactic models are shown in Figs 3, 4, and 5, In the case of the proton model, a discontinuity appears when the two spectra are added, regardless of the lower limit adopted for the extragalactic component: $10^{8} \mathrm{GeV}$ (Fig 3) or $5 \times 10^{7} \mathrm{GeV}$ (Fig 4). The latter corresponds to cosmic accelerators operating for the entire Hubble time.

For both, proton and mixed-composition models, there is a flux deficit above $10^{8} \mathrm{GeV}$. The problem is much stronger for the mixed-composition model where, regardless of the luminosity evolution of EG CR sources, the total spectrum presents a large deficit of flux between $10^{8} \mathrm{GeV}$ and $\approx 3 \times 10^{9} \mathrm{GeV}$ (Fig 5 ).

In order to solve this flux deficit, the only way out seems to be the introduction of an additional Galactic component. We estimate this component by subtracting the sum of the diffusive Galactic and extragalactic fluxes from 


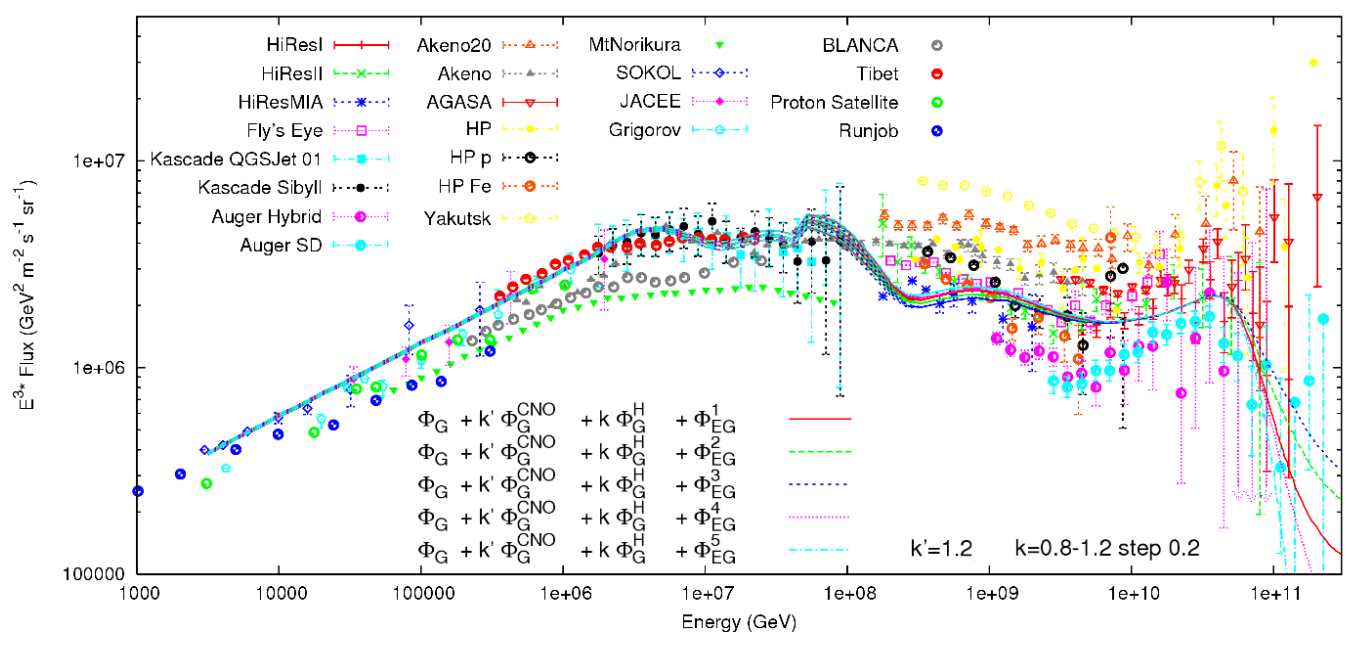

Fig. 4. Idem to Fig 3 but for the EG proton model with a lower energy limit of $5 * 10^{7} \mathrm{GeV}$.

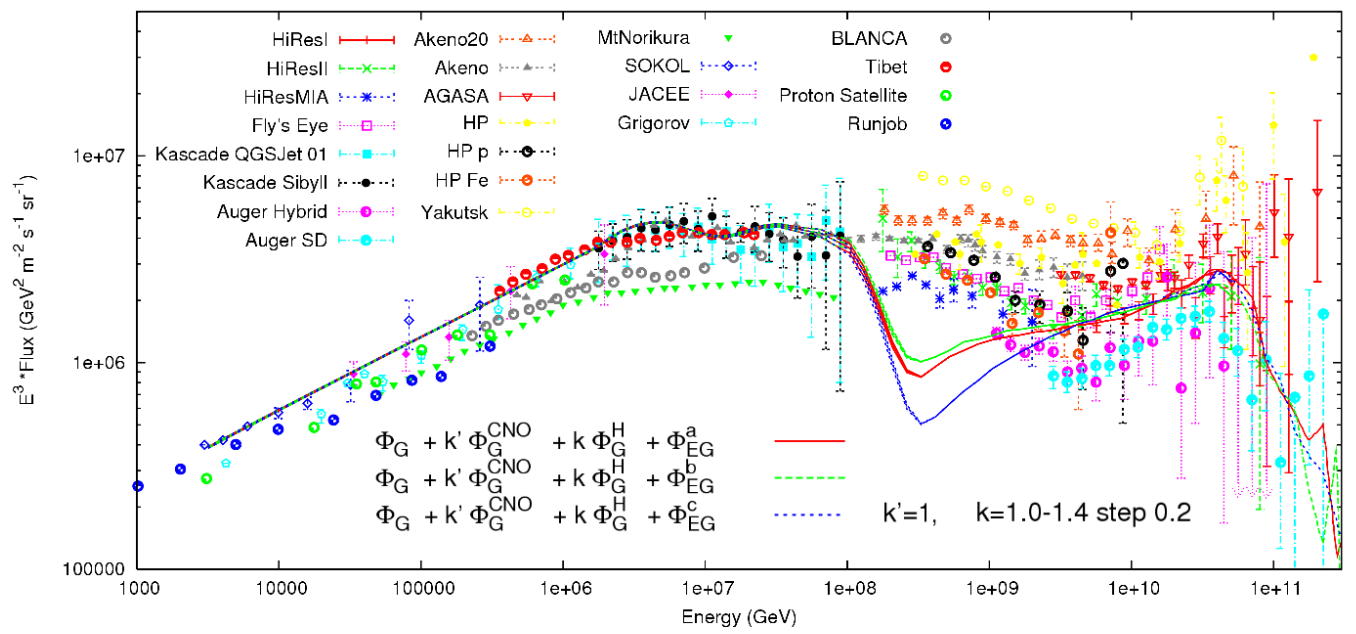

Fig. 5. Idem to Fig, 4 but for the Galactic and extragalactic spectrum for the mixedcomposition model. The CNO group $\left(\Phi_{G}^{C N O}\right)$ of the diffusive Galactic spectrum $\left(\Phi_{G}\right)$ has been renormalized by a factor 2 . Three source evolution models are considered (\$2.3): (a) strong, (b) SFR and (c) uniform.

a smooth fit to the world data. This method confirms us the need of the renormalization of the CNO group by a factor $\approx 2.2$ and $\approx 2$ for the proton and mixed-composition models, respectively. In the case of the proton models (Figs,6, 7), the observed deficit can be resolved with the introduction of an additional cosmic ray flux component (the first additional Galactic component, GA1) $\Phi_{G A 1}$. For the mixed-composition models this is not enough and one more additional cosmic ray flux component must be introduced (the second additional Galactic component, GA2) $\Phi_{G A 2}$ (Fig:8). The additional component $\Phi_{G A 1}$ common to both families of models is obtained with a shift in energy of a factor $\approx 3$ of the diffusive Galactic heavy component, renormalized by a factor $\approx 0.8$ in the case of the mixed-composition models and $\lesssim 0.6$ for the proton 


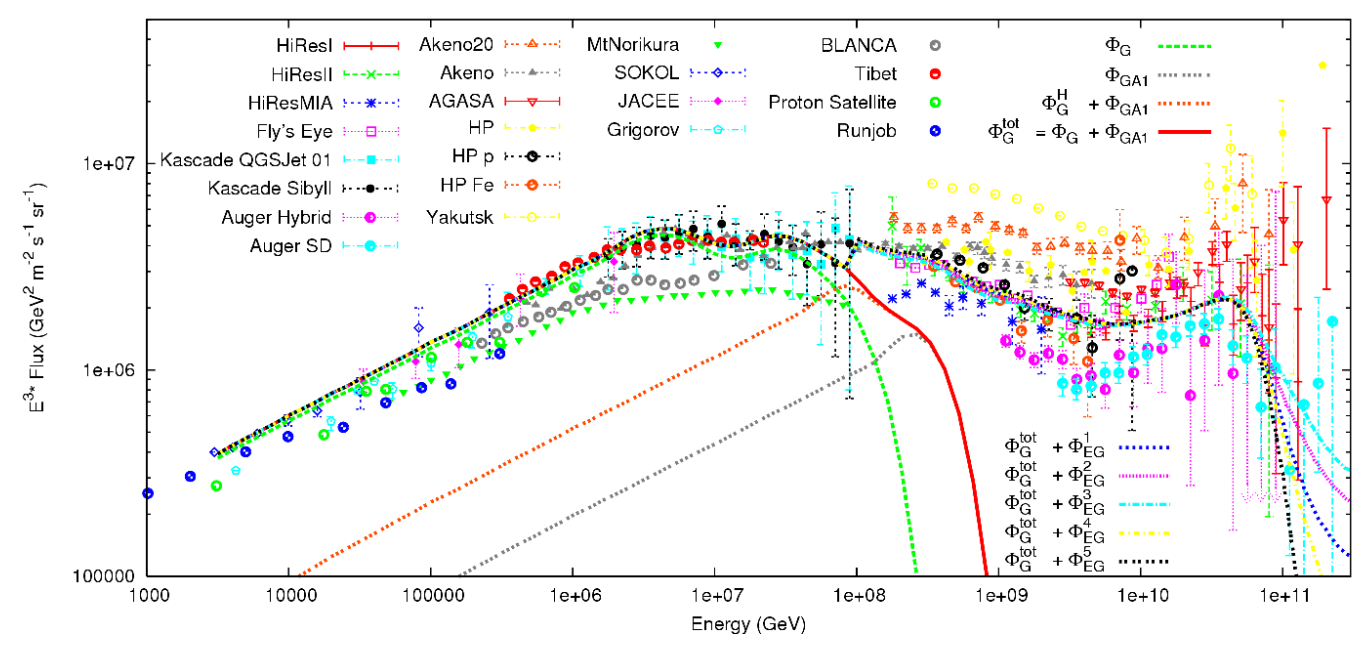

Fig. 6. Galactic and extragalactic spectrum for the proton model for an EG lower limit of $10^{8} \mathrm{GeV}$ : the additional Galactic component $\phi_{G A 1}$ and the total high energy Galactic component $\left(\phi_{G}^{H}+\phi_{G A 1}\right)$ are also shown.

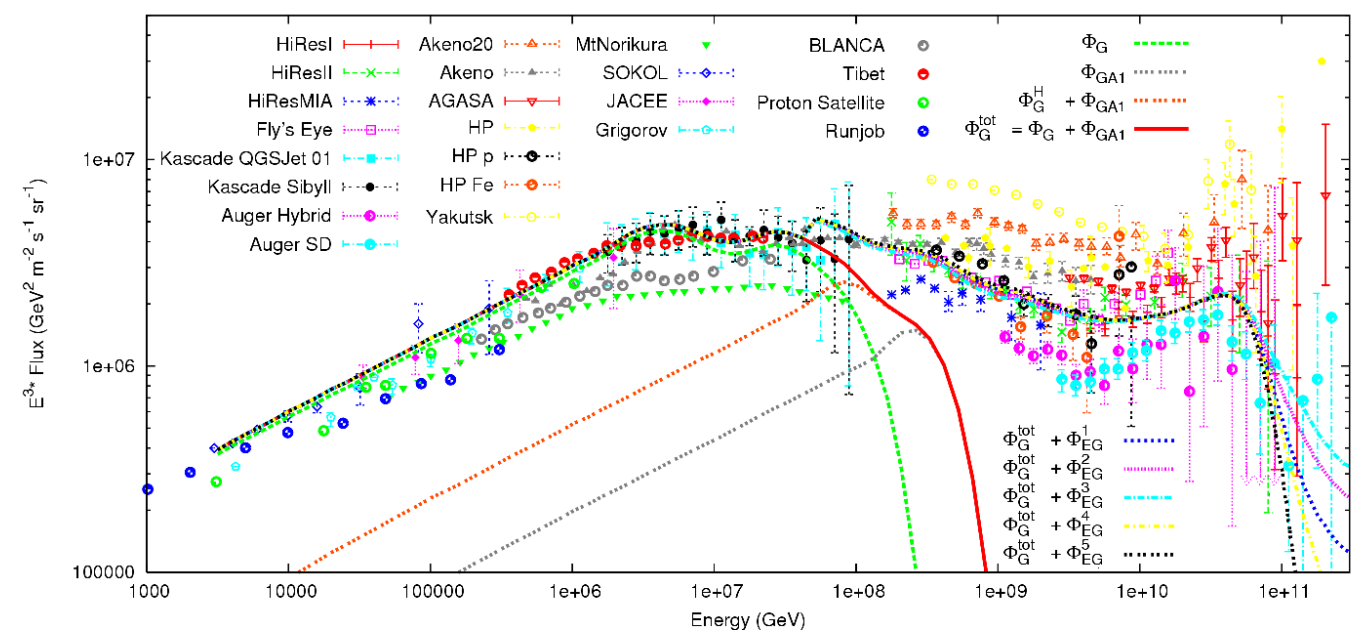

Fig. 7. Idem to Fig 6 but for the EG proton model with a lower energy limit of $5 \times 10^{7} \mathrm{GeV}$ : the additional Galactic component $\phi_{G A 1}$ and the total high energy Galactic component $\left(\phi_{G}^{H}+\phi_{G A 1}\right)$ are also shown.

models respectively. The second additional component $\Phi_{G A 2}$ is obtained in an analogous way but with an energy-shift factor of $\approx 10$ and a renormalization by a factor $\approx 0.2$. The corresponding spectra are shown in Figs 6 , 7 and 8 ,

\subsection{Discussion on the spectrum}

We have analyzed the matching conditions of the Galactic and extragalactic components of cosmic rays along the second knee and the ankle.

It seems clear that an acceptable matching of the Galactic and extragalactic fluxes can only be achieved if the Galaxy has additional accelerators, besides 


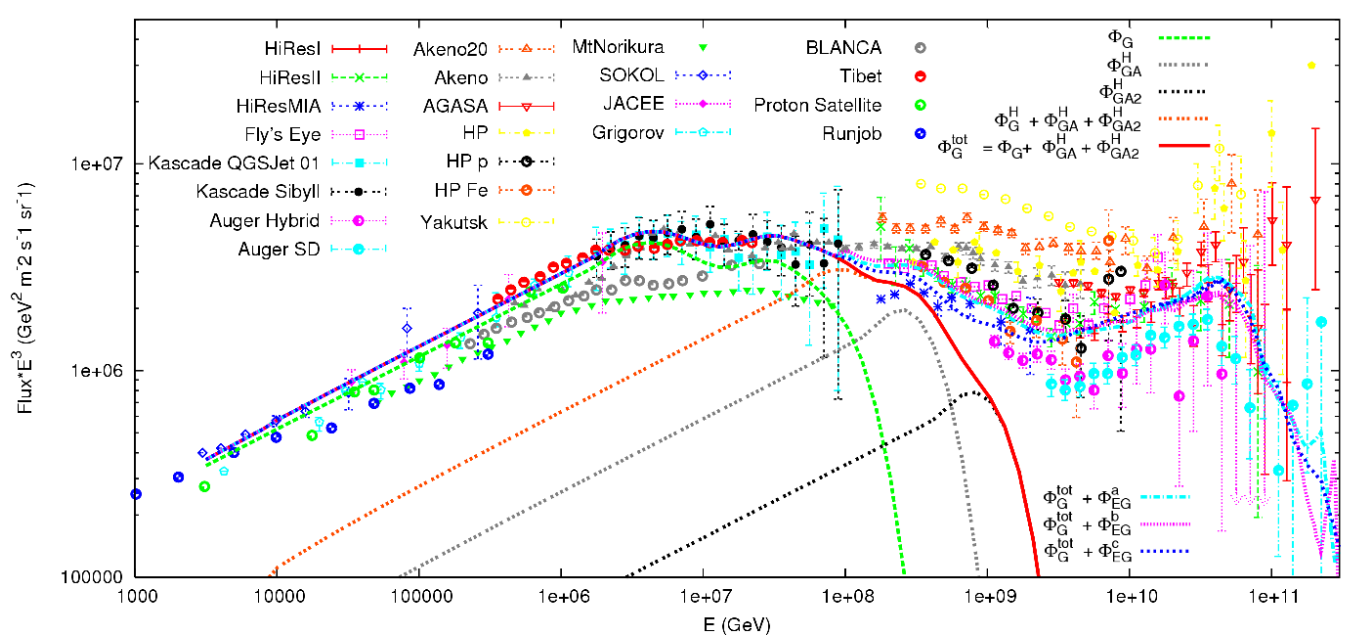

Fig. 8. Galactic and extragalactic spectrum for the mixed-composition model: the two additional components $\phi_{G A 1}, \phi_{G A 2}$ and the total high energy Galactic component $\left(\phi_{G}^{H}+\phi_{G A 1}+\phi_{G A 2}\right)$ are shown.

the fiducial SNRs assumed here, operating in the interstellar medium.

In the particular case of the proton model, only one additional component is required. This could well represent the contribution from compact and highly magnetized SNRs, like those occurring in the central, high density regions of the Galactic bulge, inside the dense cores of molecular clouds or those expanding into the circumstellar winds of their progenitors. Actually, this component does not need to originate in a particular type of source in itself, but could be the result of a non-homogeneous SNR population drawn from a spectrum of progenitor masses and evolving in different environments corresponding to the various gas phases that fill the interstellar medium [70]. From the point of view of CR luminosity this should not be a problem, since the Fe component and the additional high energy component amount to $\sim 9 \%$ and $\lesssim 1 \%$, respectively, of the total Galactic diffusive spectrum.

In the specific case of the proton model, it is apparent that a perfectly smooth match between the Galactic and the extragalactic spectra is not possible. Some discontinuity or wiggle seems unavoidable in the energy spectrum between $\sim 3 \times 10^{16}$ and $\sim 10^{17} \mathrm{eV}$ (see figures [3, 4, 6] and 7). This is due to the fact that the extragalactic spectrum should have a rather abrupt low energy cut-off due to the finite distance to the nearest extragalactic sources and their limited age. Of course, whether such spectral feature is actually observable is strongly dependent in practice on the magnitude of the experimental errors in the determination of the primary energy from shower measurements and on the available statistics.

The matching of the mixed-composition model, on the other hand, has wider 
astrophysical implications for the Galaxy. The Galactic CR production has to be extended up to at least the middle of the dip and this requires, besides the previous additional component, another high energy Galactic component. The origin of these cosmic rays pushes even further the acceleration requirements imposed on the Galaxy. It is likely that a different population of Galactic accelerators must be invoked. Viable candidates could include, for example, rapidly spinning inductors, like pulsars or magnetars, or even very high energy episodic events like Galactic gamma ray bursts [71]. If this were the case, it is very likely that photon emission at $\mathrm{TeV}$ energies should uncover the sources. Changes in propagation regime inside the Galaxy at these high energies should manifest as an anisotropic component embedded in the isotropic incoming extragalactic background. Again, considerable statistics might be necessary to make such effect observable.

The energy requirements involved in the production of the second additional component are rather modest: $\sim 10^{-7}$ of the SNR CR component, or $\sim 10^{34}$ erg/sec pumped into particles between $\sim 10^{17}$ and $\sim 10^{18} \mathrm{eV}$. Therefore, few, or even a single source, could be responsible for this component. This carries attached the potential problem of undesirable fine-tuning because of the requirement that, at this precise moment in time, the CR luminosity of these few (or this single) sources is such that it allows for a smooth spectral matching along the ankle.

It is important to note that the region between some few times $10^{17} \mathrm{eV}$ and approximately $10^{19} \mathrm{eV}$ is a transition region from the point of view of propagation [172. In fact, Larmor radius (in pc) of a CR nucleus of charge $Z$ can be conveniently parametrized as:

$$
r_{L, p c} \sim \frac{10}{Z} \times\left(\frac{E_{E e V}}{B_{\mu G}}\right)
$$

where $E_{E e V}$ is its energy and $B_{\mu G}$ is some appropriate average of the Galactic magnetic field inside the propagation region. Since the transversal dimensions of the Galactic disk are on the order of a few times $10^{2} \mathrm{pc}$, we see that the diffusion approximation for protons should start to be broken somewhere between the second knee and $1 \mathrm{EeV}$. The same should happen to heavier nuclei at progressively higher total energies: $\sim 3 \mathrm{EeV}$ for the CNO group and $\sim 10$ $\mathrm{EeV}$ for Fe. The corresponding transition for each nuclei should be gradual, with the propagation eventually becoming ballistic at higher energies. This transition results in a complicated picture in which the end of the Galactic confinement spans almost 1.5 decade in energy, depending on the particular nuclei considered. Our interpretation of the additional Galactic components (but not their necessity) is affected to some extent by the implicit assumption that the particles propagate diffusively. This assumption is increasingly ques- 
tionable for protons as we approach $1 \mathrm{EeV}$, but should be valid for heavier nuclei in the energy range considered here. We are currently working on a detailed analysis of these effects which will be presented elsewhere.

\section{Composition}

The different extragalactic models are able to produce total spectra that are indistinguishable within the current experimental resolution. The composition of UHECR is essential to understand the transition between Galactic and extragalactic cosmic rays and to discriminate the different astrophysical scenarios.

Since UHECR experiments do not directly measure the composition, we have to infer it from parameters characterizing the shower development profile at ground. One of the most reliable parameters is $X_{\max }$, the atmospheric depth of the maximum longitudinal development of a shower [73]. The variation of $\left\langle X_{\max }\right\rangle$ with energy gives information about the change in composition of the CR flux.

Different hadronic interaction models (HIMs) can be used to interpret the $X_{\max }$ dependence on energy and on primary composition, and this is the main uncertainty associated with this parameter. In this section we will use different parameterizations of $\left\langle X_{\max }\right\rangle$ deduced from the hadronic interaction models currently in use, in order to infer the composition energy profile for the different cases of Galactic-extragalactic combined spectrum described in section $\$ 2.4$.

\subsection{Hadronic interaction models}

The composition predictions depend on the HIM used. For the sake of completeness, we take into account in what follows all the HIMs currently used in the literature:

- EPOS 1.6

- QGSJet 01

- QGSJetII v02

- QGSJetII v03

- Sibyll 2.1

For each hadronic interaction model, we calculate a parameterization of $\left\langle X_{\max }\right\rangle$ as a function of the hadronic interaction model and of the primary energy and composition: 
Table 1

Coefficients of the parameterization of $\left\langle X_{\max }\right\rangle$ as a function of primary energy and composition (eq. 5) for different hadronic interaction models.

\begin{tabular}{|c|r|r|r|r|r|r|}
\hline Model & $a$ & $b$ & $c$ & $p$ & $\alpha$ & $\beta$ \\
\hline QGSJetII-v03 & -0.033 & -0.010 & 58.313 & 184.315 & $9.60 \times 10^{-4}$ & $-2.41 \times 10^{-3}$ \\
QGSJetII-v02 & 0.184 & -5.930 & 115.111 & -15.895 & $2.00 \times 10^{-3}$ & $6.50 \times 10^{-2}$ \\
QGSJet01 & 0.074 & -3.695 & 103.804 & -19.123 & $6.85 \times 10^{-4}$ & $2.14 \times 10^{-2}$ \\
EPOS 1.6 & -0.011 & 1.831 & 29.212 & 265.847 & $-1.47 \times 10^{-4}$ & $4.69 \times 10^{-4}$ \\
Sibyll 2.1 & 0.300 & -8.422 & 134.960 & -72.051 & $3.38 \times 10^{-4}$ & $2.62 \times 10^{-3}$ \\
\hline
\end{tabular}

$$
\begin{aligned}
\left\langle X_{\max }\right\rangle(E, A, i)= & {\left[a_{i}\left(\log \frac{E}{A \epsilon}\right)^{2}+b_{i} \log \frac{E}{A \epsilon}+c_{i}\right]\left(1+\alpha_{i} A\right) } \\
& +p_{i}\left(1+\beta_{i} A\right),
\end{aligned}
$$

where $E$ is the primary energy, $A$ is the atomic mass of the primary CR, $i$ is the hadronic interaction model, $\epsilon \sim 81 \mathrm{MeV}$ is the critical energy in air and $a_{i}, b_{i}, c_{i}, p_{i}, \alpha_{i}, \beta_{i}$ are coefficients determined by fitting equation 5 to shower simulations (see Table 1).

Parameterization 5 is a good approximation in the energy range $\left[10^{17} \mathrm{eV}, 10^{20} \mathrm{eV}\right]$. Our parameterization for $\mathrm{p}$ and Fe, for several HIMs, is shown in Fig 9 together with the experimental results of HiRes [74], HiResMIA [53], Fly's Eye [75] and Auger [76].

This parameterization allows us to compute the average $X_{\max }$ for showers generated by any nucleus of interest and for all observed primary energies above the second knee.

With this parameterization, in the following sections we reproduce $\left\langle X_{\max }\right\rangle$ energy profiles for different composition scenarios in the context of the various cases of the combined Galactic-extragalactic spectrum.

\subsection{Galactic-extragalactic combined spectra: $X_{\max }$ energy profiles}

Heretofore, we have analyzed from the point of view of the energy spectrum, different scenarios for the combination of the Galactic flux with alternative extragalactic models. For each of these scenarios, and under different assumptions for the composition of individual flux components, we estimate $X_{\max }$ along the transition region. 


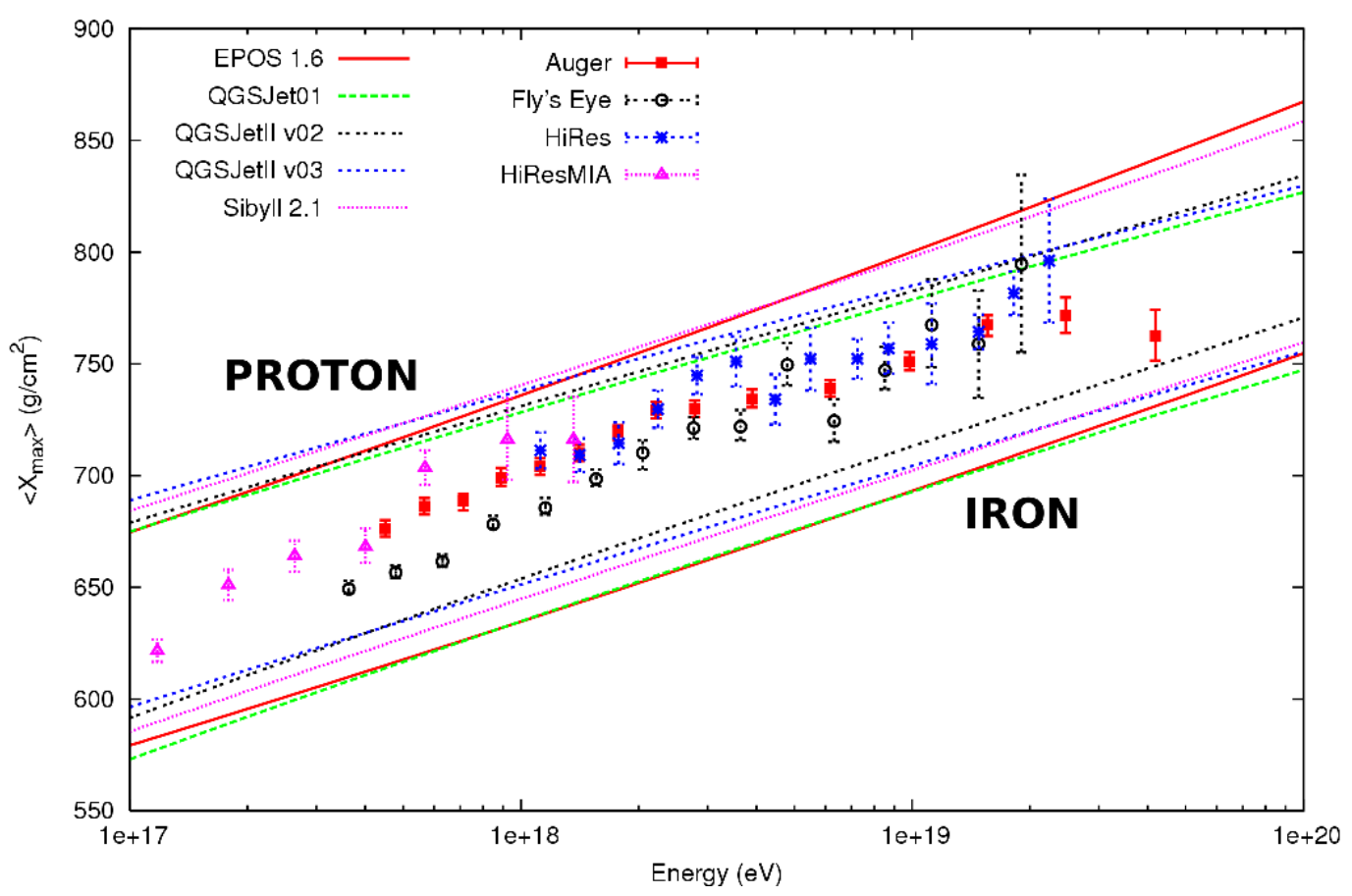

Fig. 9. $\left\langle X_{\max }\right\rangle$ parameterization as a function of primary energy and composition. For each HIM, the $\left\langle X_{\max }\right\rangle$ dependence on energy for proton and Iron primary is shown. Experimental data from HiRes, HiResMIA, Fly's Eye and Auger are also shown.

As seen in $\$ 2.4$, an acceptable matching of the Galactic and extragalactic fluxes can only be achieved if the Galaxy has additional accelerators besides the regular SNRs assumed in \$2.1. This can be achieved by including either one (pure proton EG spectrum) or two (mixed-composition EG spectrum) additional Galactic components.

In order to infer $X_{\max }$ for the combined flux, we have to do some assumptions on the composition of the additional Galactic components.

The first additional Galactic component (GA1), which is needed in both of the EG scenarios previously considered, is probably contributed by compact, highly magnetized SNRs. Consequently this component is likely to be dominated by heavy elements, say, Iron, at the highest energies.

The second additional Galactic component (GA2), on the other hand, might be associated with a minor population of still more powerful SNR accelerators or with a completely different population of particle accelerators, e.g., rapidly spinning inductors, like pulsars or magnetars. In the first case, GA2 would be Fe dominated, while in the latter case, GA2 could be proton dominated. Consequently, we consider two different possibilities for GA2: pure proton and pure Fe. 


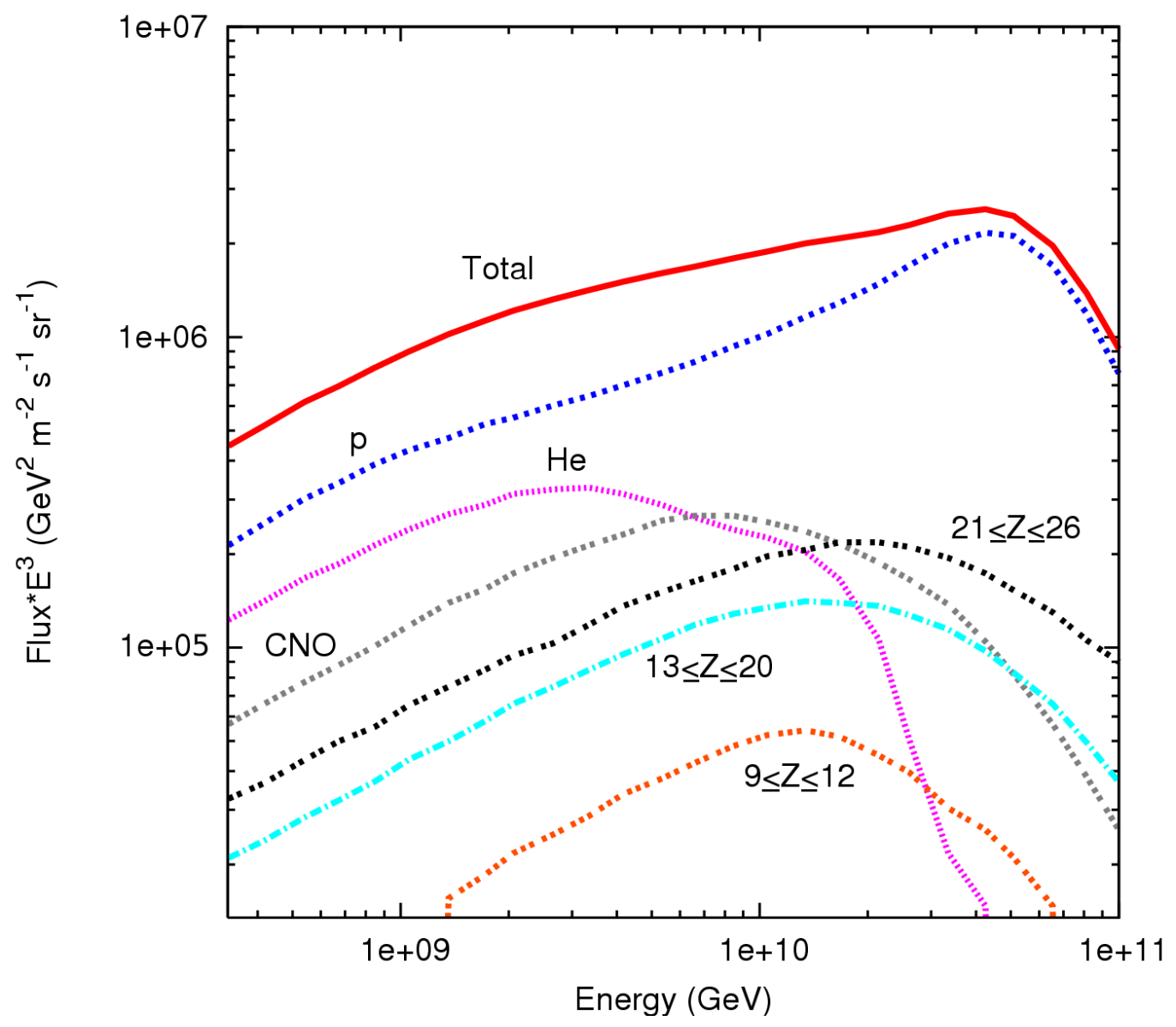

Fig. 10. Mixed-composition EG scenario of Allard and co-workers. Contribution to the extragalactic flux of different nuclei in the "uniform source distribution model". (Adapted from: [25]).

We calculate $X_{\max }$ as a function of energy in the energy range $\left[10^{17} \mathrm{eV}, 10^{20} \mathrm{eV}\right]$ for all the possible combination of Galactic and extragalactic spectra previously discussed in $\$ 2.4$ from the point of view of the shape of the energy spectrum 2:

- pure proton EG model: we consider the combination of the calculated Galactic spectrum from SNRs with the EG "universal" (see \$2.3) spectrum in the two cases of lower EG energy limit $\left(5 \times 10^{16} \mathrm{eV}\right.$ and $\left.\sim 10^{17} \mathrm{eV}\right)$; the composition of the Galactic additional component is assumed to be heavy (Iron);

- mixed EG composition model: we consider the combination of the calculated Galactic spectrum from SNRs with the mixed EG spectrum in its "uniform source distribution model" variant (see \$2.3); we also assume that GA1 is composed by pure Fe, while we analyze two opposite scenarios for GA2: pure proton and pure Fe. The EG composition is taken from Fig 10 [25].

$\overline{2}$ For the diffusive Galactic spectrum from SNRs we kept the renormalization of the CNO group by a factor $\sim 2$ determined in the previous analysis ( 92.4 ) 


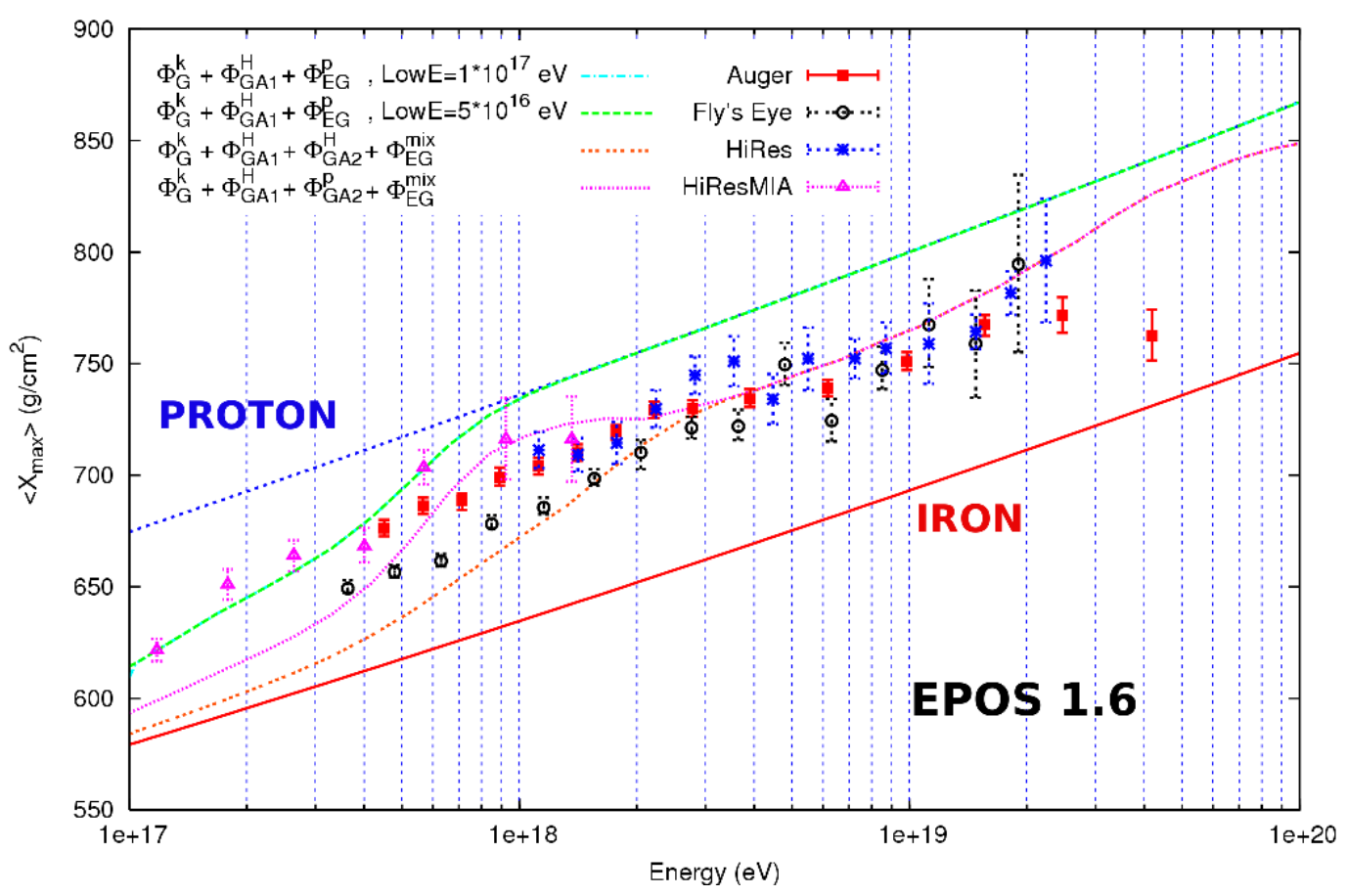

Fig. 11. $X_{\max }$, EPOS 1.6: the $X_{\max }$ profiles calculated for all the possible combined spectra are shown superimposed onto the experimental data. The curves represent: (i) the combined spectra for an EG proton model with lower energy limit $\sim 10^{17} \mathrm{eV}$ (cyan blue) and $5 \times 10^{17} \mathrm{eV}$ (green) respectively; (ii) the combined spectra for an EG mixed-composition model with pure proton (purple) and pure Fe (brown-orange) GA2. $\left\langle X_{\max }\right\rangle$ values are calculated using the EPOS 1.6 HIM.

For all these scenarios with different component spectra and composition assumptions, we calculate $\left\langle X_{\max }\right\rangle$ values in the energy range $\left[10^{17} \mathrm{eV}, 10^{20} \mathrm{eV}\right]$ using the parameterization given in $\$ 3.1$ for the various HIMs in current use.

The results are shown for each HIM in Figs,11, 12, 13, 14, and 15, together with the experimental results of HiRes [74], HiResMIA [53], Fly's Eye [75] and Auger [76].

First of all we notice that, as expected, for all the HIMs the theoretical $X_{\max }$ values in the case of EG proton model are indistinguishable for the two different EG lower energy limits (green and cyan blue curves are superimposed on the figures). On the other hand, the model is very much compatible with HiRes-MIA data below $10^{18} \mathrm{eV}$ while, at higher energies, $\left\langle X_{\max }\right\rangle$ is too large when compared to any of the available data sets.

As expected, much more variability in the calculated elongation rates is observed for the lower energy regime in the case of EG mixed-composition models, depending on the assumptions made about the composition of GA2 (purple and brown-orange curves). However, at energies larger than $\sim 3 \times 10^{18} \mathrm{eV}$, all the solutions converge to the same profile since the effects of GA2 become 


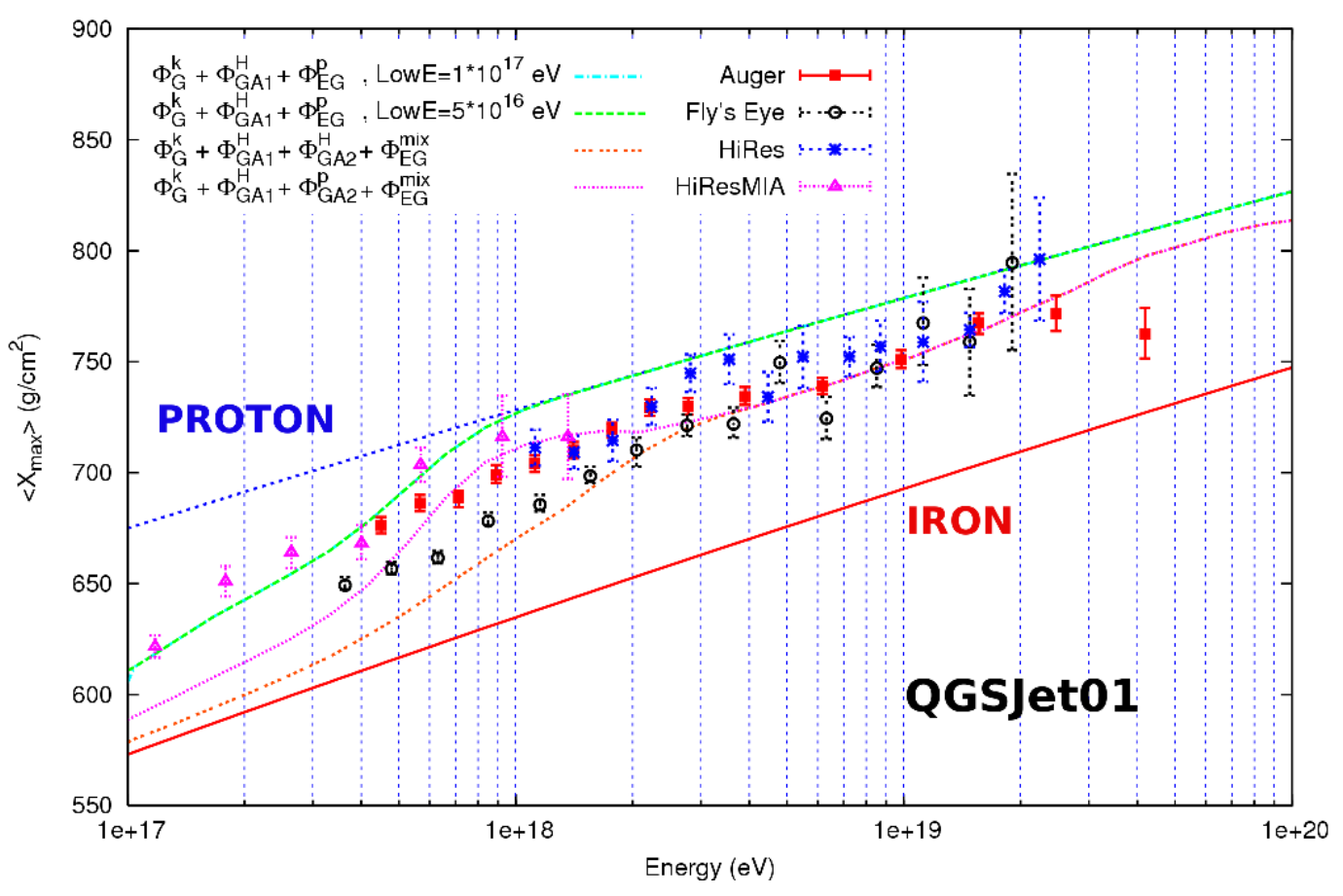

Fig. 12. $X_{\max }$, QGSJet01: idem to Fig[11 but using the QGSJet01 HIM.

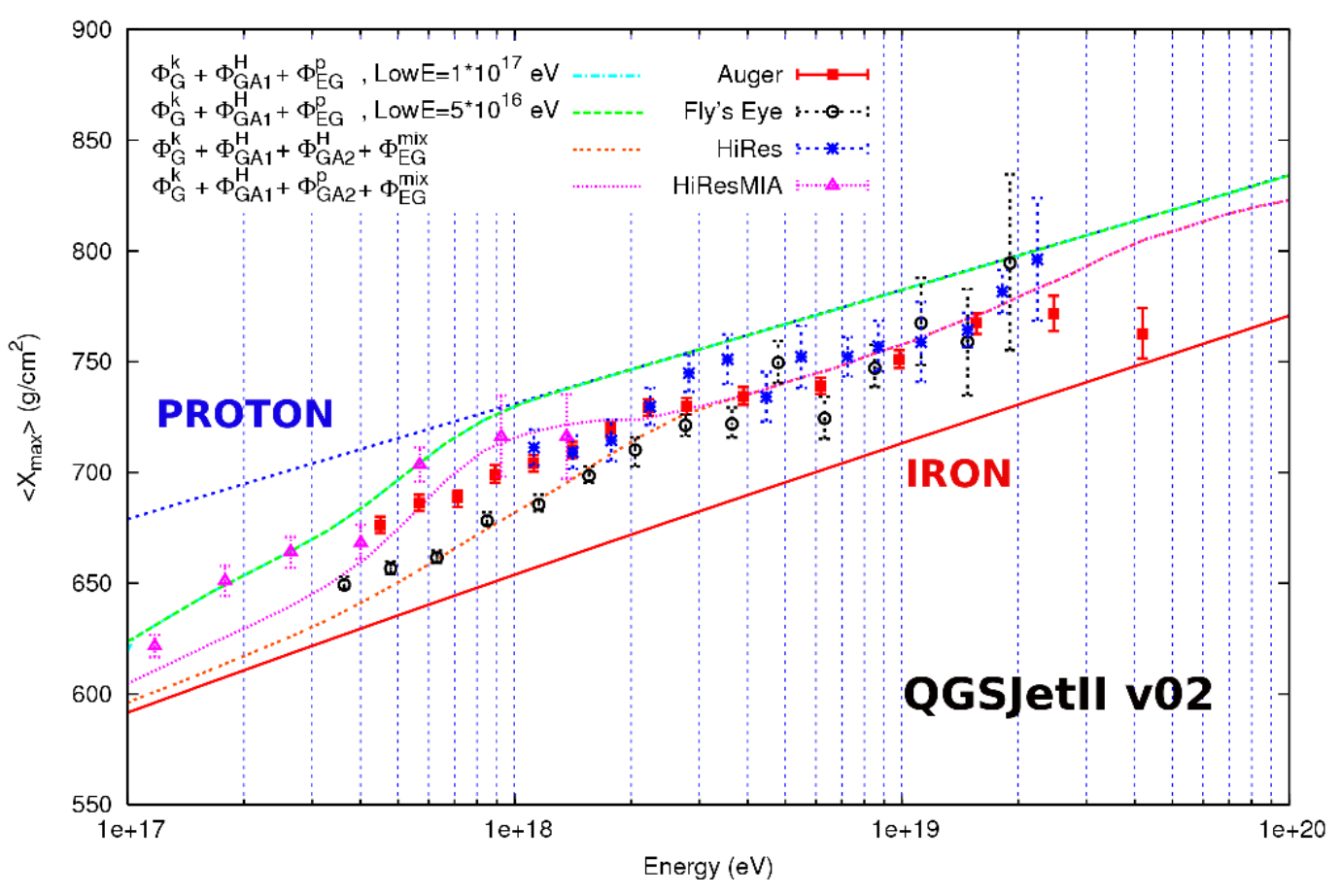

Fig. 13. $X_{\max }$, QGSJetII v02: idem to Fig 11 but using the QGSJetII v02 HIM.

progressively negligible on the combined composition profile. All these results are qualitatively independent of the assumed HIM. Nevertheless, even if all the HIMs give basically the same trend at all energies, actually what data set is quantitatively compatible with the mixed composition theoretical models 


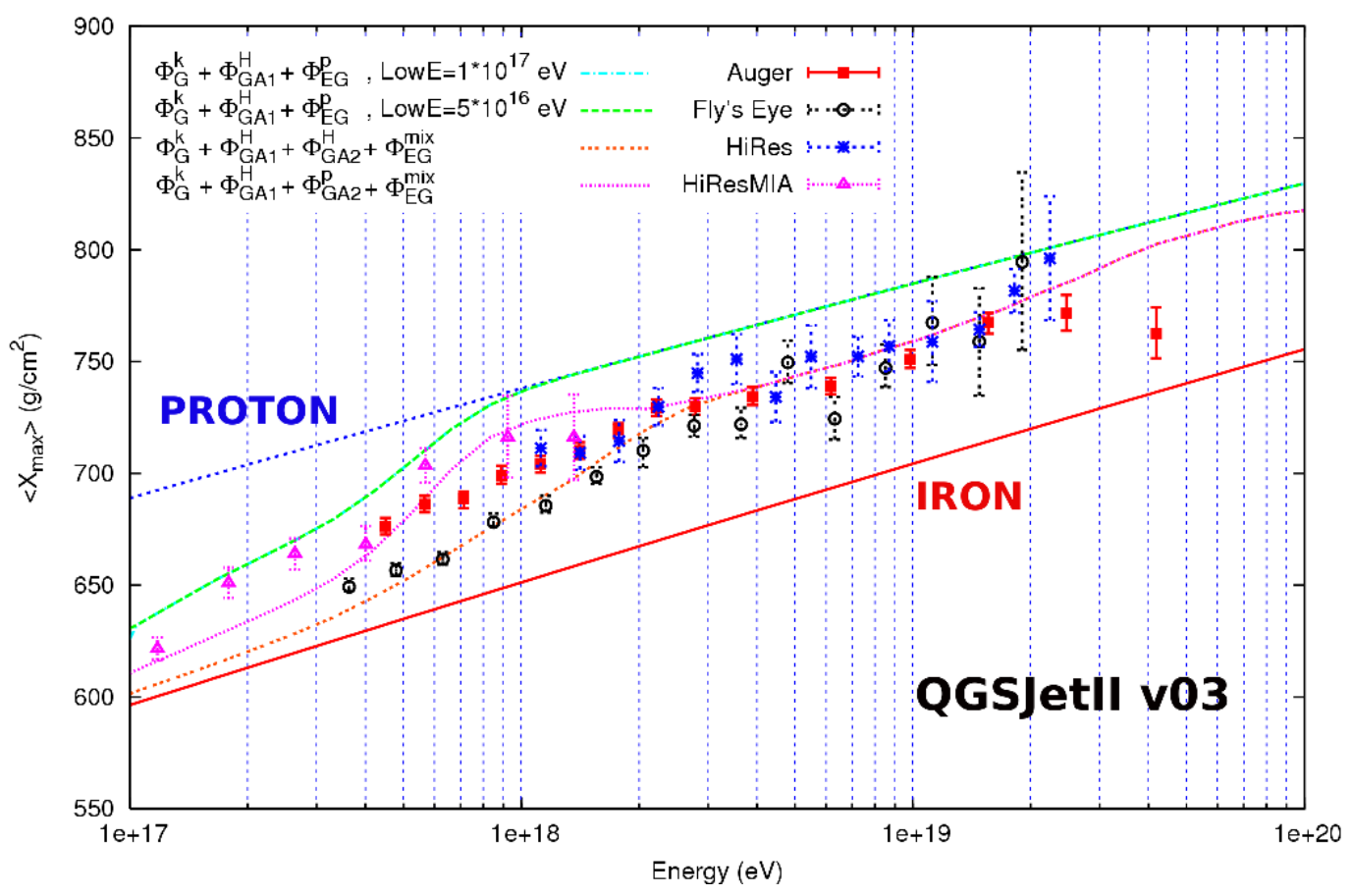

Fig. 14. $X_{\max }$, QGSJetII v03: idem to Fig[11 but using the QGSJetII v03 HIM.

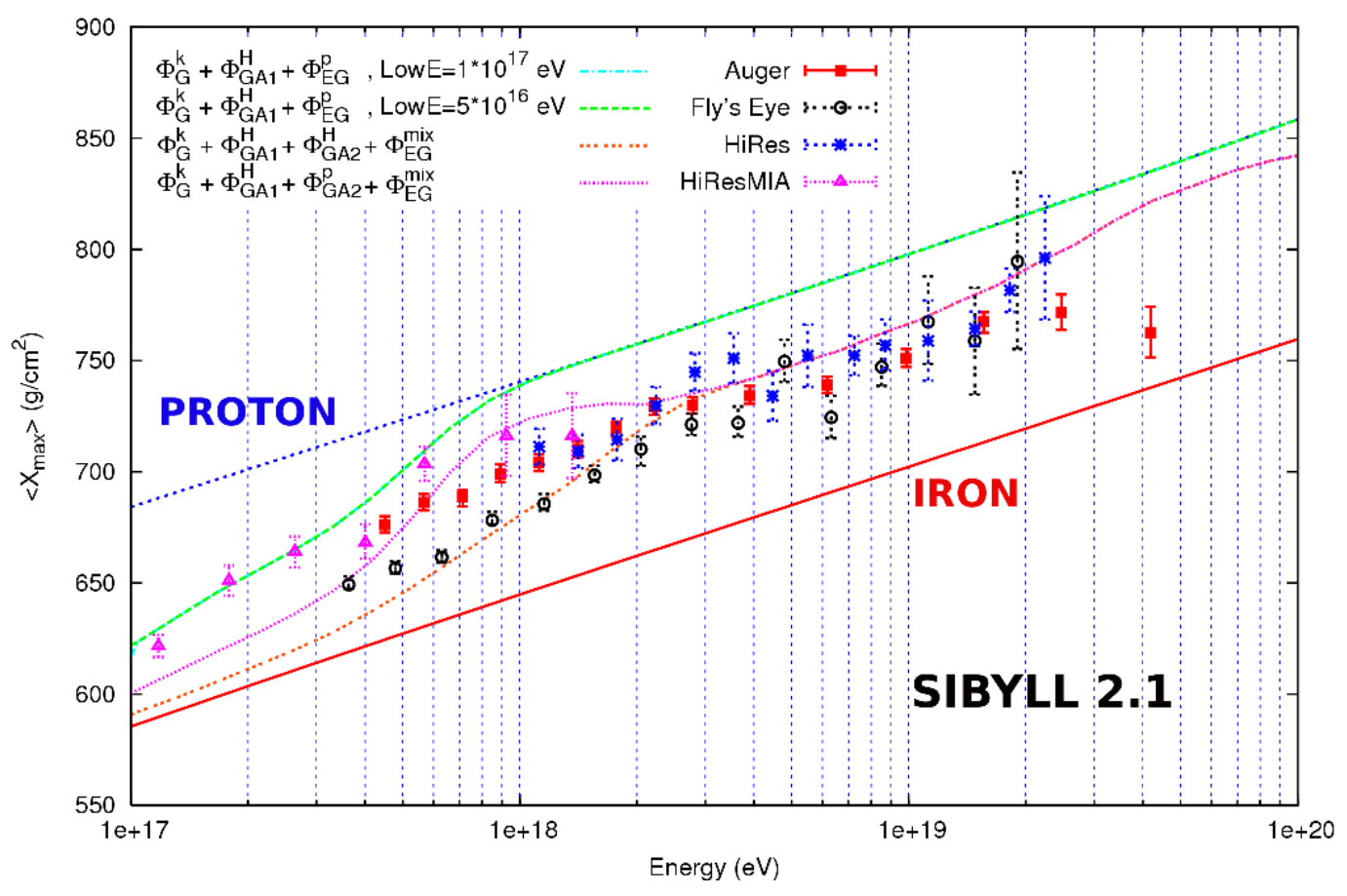

Fig. 15. $X_{\max }$, Sibyll 2.1: idem to Fig 11 but using the Sibyll 2.1 HIM.

at energies beyond $3 \times 10^{18} \mathrm{eV}$ does depend on the assumed HIM. Furthermore, at the highest energies, $>10^{19} \mathrm{eV}$, there could be an indication that the models give a systematically lighter composition than what the data suggests. Unfortunately, at present, statistics at these energies are too low for all 
experiments as to render any solid conclusion.

The most critical energy region for the understanding of the Galactic-extragalactic transition and disentangling its flux components, is $\sim 10^{17}-\sim 3 \times 10^{18} \mathrm{eV}$. Inside this region, different experimental results, and they are different indeed, imply very different astrophysical scenarios, in particular with regard to the highest accelerators present in our own Galaxy.

In the following sections we will center on two of the most important experimental results today, those of Auger and HiRes, and test what, if any, further modifications should be applied to the composition profile in order to make the data and the theoretical models as compatible as possible.

\subsection{Composition evolution along the transition region}

If our understanding of $\left\langle X_{\max }\right\rangle$ in terms of composition is reasonably correct, Auger and HiRes elongation rate data suggest a composition profile along the ankle compatible with a mixed extragalactic composition, despite differences in the energy dependence of $X_{\max }$ for both experiments.

The agreement between Auger $X_{\max }$ data [76] and our predictions is remarkable at energies around $3 \times 10^{18} \mathrm{eV}$ for all HIMs. This agreement extends to higher energies, even beyond $2 \times 10^{19} \mathrm{eV}$, for QGSJetI and QGSJetII v02 and v03, while EPOS and SIBYLL display different trends. The picture is more complicated at lower energy where, under the present assumptions, there is no agreement regardless of the assumed HIM.

The situation almost reverses in the case of the HiRes-MIA combined data set, for which a good agreement can be obtained at low energies with the EG proton model, but there is no clear fit at higher energies.

It must be noted, however, that the composition profiles shown in Figs 11, 12, 13, 14 and 15, result simply from the combination of our previous solution to the total energy spectrum for the mixed model and the unmodified mixed model compositions as determined by [25], plus fixed compositions for GA1 and GA2.

Therefore, in this section we analyze whether the composition profile of the two additional Galactic components and of the extragalactic flux can be modified in a suitable way in order to satisfy, simultaneously, the existing spectral and elongation rate data along the transition region.

We use to this end a simplified model in which the shape of the extragalactic energy spectrum is kept, to first order, identical to Allard's mixed composition 
model [3], but the composition is limited to just two nuclei, proton and Iron, whose relative abundances can change appropriately in order to reproduce the behavior of $\left\langle X_{\max }\right\rangle$ as a function of energy. The admitted lack of consistency in this approach is, we believe, compensated by the insight gained into the phenomenological constraints imposed by the data on the astrophysical models at play 3 .

On the other hand, the spectral shapes of both additional components and their normalizations are preserved, but their compositions as a function of energy are now functions to be determined during the fitting process. A binary mixture of $\mathrm{p}$ and Fe is also assumed.

We further assume, a priori, that the diffusive Galactic spectrum and its composition are the ones determined previously in $\$ 2.4$ with the calculated renormalization of the CNO group. The diffusive Galactic flux from SNRs, spectrum as well as composition, is kept constant afterward during the fitting procedure.

We apply this procedure to both, Auger and HiRes data in order to gain an insight on how present experimental uncertainties can affect our astrophysical understanding of the transition region.

\subsubsection{Auger data}

Fig.16 shows the total spectrum and Galactic components that are used in this section. For each source evolution model (see \$2.3), we renormalize the EG spectrum to the surface detector (SD) Auger flux at $10^{19.05} \mathrm{eV}$ [58], where one can expect a negligible contribution from the Galactic CR flux. Afterward, we renormalize the GA1 and GA2 in order to match the total spectrum with the Auger spectrum data; the hybrid Auger spectrum 4 at lower energy, $10^{18}-10^{18.3} \mathrm{eV}$ [19], is used to this end. The lowest energy branch of the ankle is approximated by a smooth interpolation between the highest energy KASCADE spectrum and the lowest energy hybrid Auger data.

It must be noted that, regardless of the renormalization, the extragalactic theoretical spectral models are too soft to allow a good fit to the Auger data at the highest energies. This inconsistency have also effects at lower energies, where the position of the ankle is artificially pushed down from the position determined by Auger at $\sim 10^{18.6} \mathrm{eV}$. Therefore, as a compromise, we consider

3 An alternative analysis to the spectral fit is the use of slanted shower data which, for Akeno data was performed by [70].

4 The Hybrid technique consists in reconstructing the CR showers using the complementary information given by the two independent detectors, the fluorescence detector (FD) and the surface detector (SD). These data allow the reconstruction of the cosmic ray energy spectrum up to energies below the mid-ankle. 


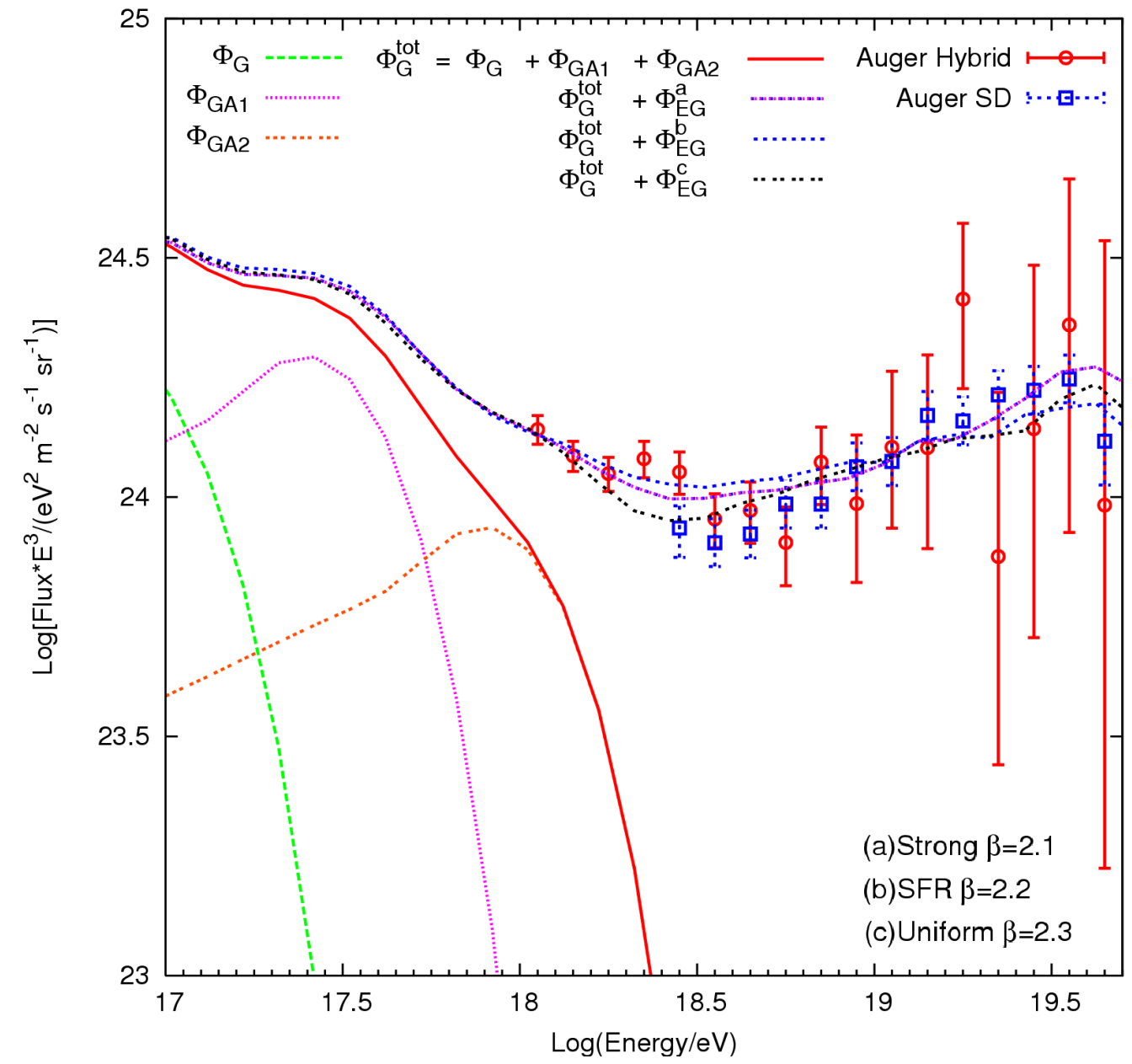

Fig. 16. Combined total spectrum $\Phi_{G}^{t o t}+\Phi_{E G}^{a, b, c}$ renormalized to Auger spectrum data for different EG evolution models (\$2.3). The Galactic spectrum $\Phi_{G}$, the Galactic additional components $\Phi_{G A 1}, \Phi_{G A 2}$ and the total Galactic spectrum $\Phi_{G}^{\text {tot }}$ are also shown. The normalization of the second Galactic additional component is the one determined for the EG "uniform source distribution" model. See the text for details.

here the model corresponding to a "uniform source distribution", which is the hardest one and provides the best visual fit to the highest energy data.

From the estimated combined Galactic-extragalactic total spectrum and using the $X_{\max }$ parameterization given in $\$ 3.1$, eq. 5, we compute the relative abundances of proton and Fe nuclei of the EG spectrum and of the two additional Galactic components (GA1 and GA2) that match the Auger $\left\langle X_{\max }\right\rangle$ data. The EG, GA1 and GA2 compositions are determined in the energy range of available Auger $X_{\max }$ data, $\sim 3 \times 10^{17}$ to $\sim 5 \times 10^{19}$, for all the hadronic interaction models.

The corresponding Iron and proton fluxes for the EG spectrum and for GA1 and GA2 are shown in Fig,17. The different curves for each nucleus correspond to inferences obtained with different HIMs. 

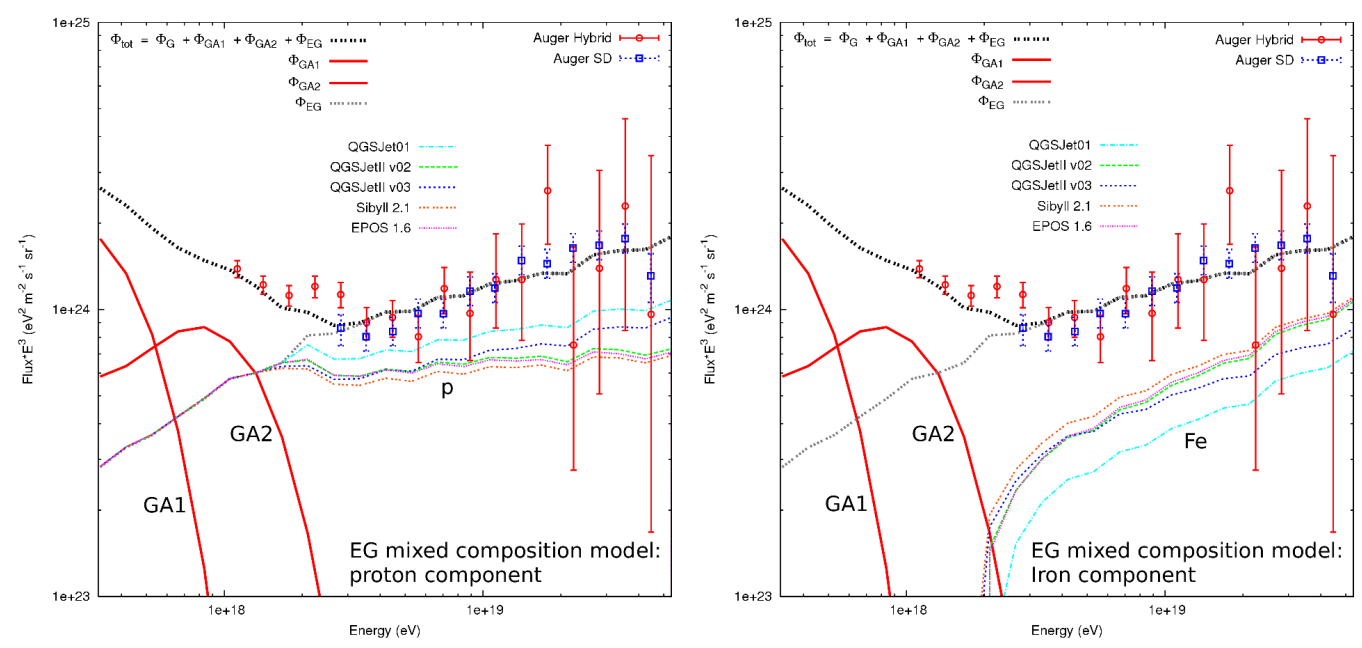

(a) EG composition: proton (left) and Iron (right) components.
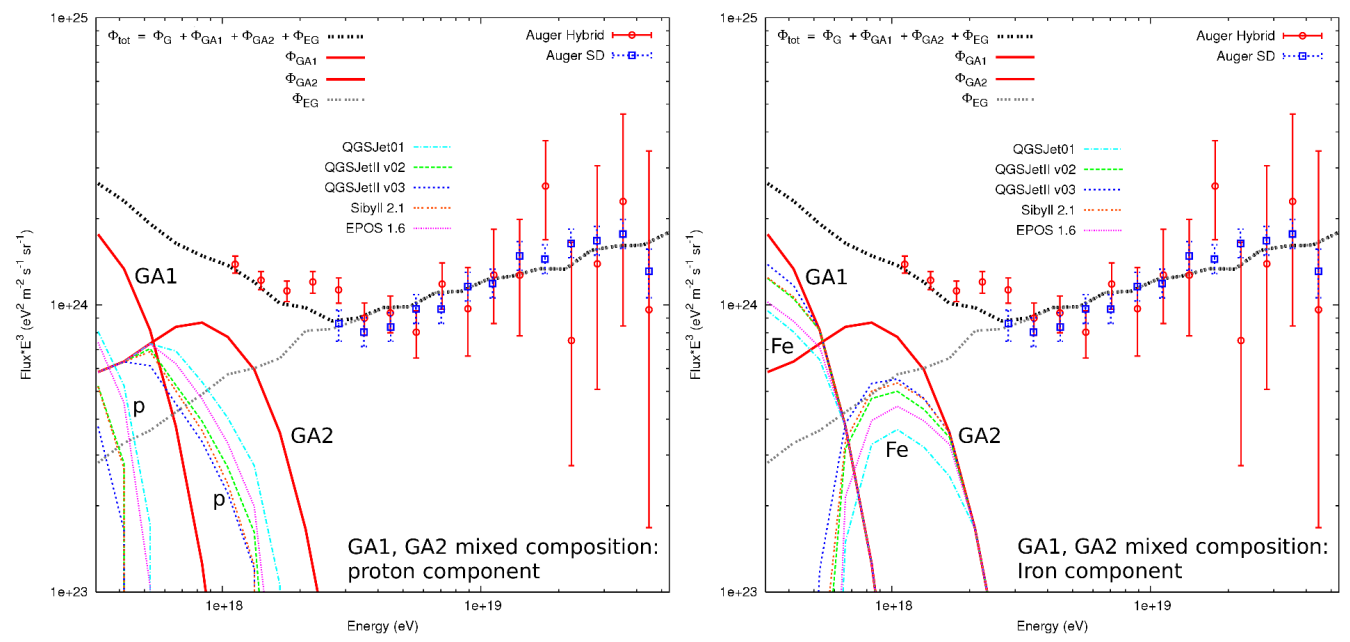

(b) GA1 and GA2 composition: proton (left) and Iron (right) components.

Fig. 17. Auger data. (a) EG proton and Iron composition; (b) GA1 and GA2 proton and Iron compositions. The fluxes are calculated, for each HIM, in order to reproduce the Auger $\left\langle X_{\max }\right\rangle$ data. The Galactic additional components $\left(\Phi_{G A 1}, \Phi_{G A 2}\right)$, the EG spectrum $\left(\Phi_{E G}\right)$ and the total spectrum $\left(\Phi_{t o t}=\Phi_{G}+\Phi_{G A 1}+\Phi_{G A 2}+\Phi_{E G}\right)$ are also shown, as well as the Auger spectrum data.

The corresponding average atomic weights, $\langle A\rangle$, for each HIM are shown in Fig,20,

\subsubsection{HiRes data}

In order to assess the astrophysical implications of the present experimental uncertainties in the total spectrum and elongation rate, we also apply here the same procedure as in the previous section to the HiRes data [74,52,53]. Fig. 18 shows the total spectrum, for different assumptions regarding the redshift evolution of the sources, and the Galactic components under consideration. 


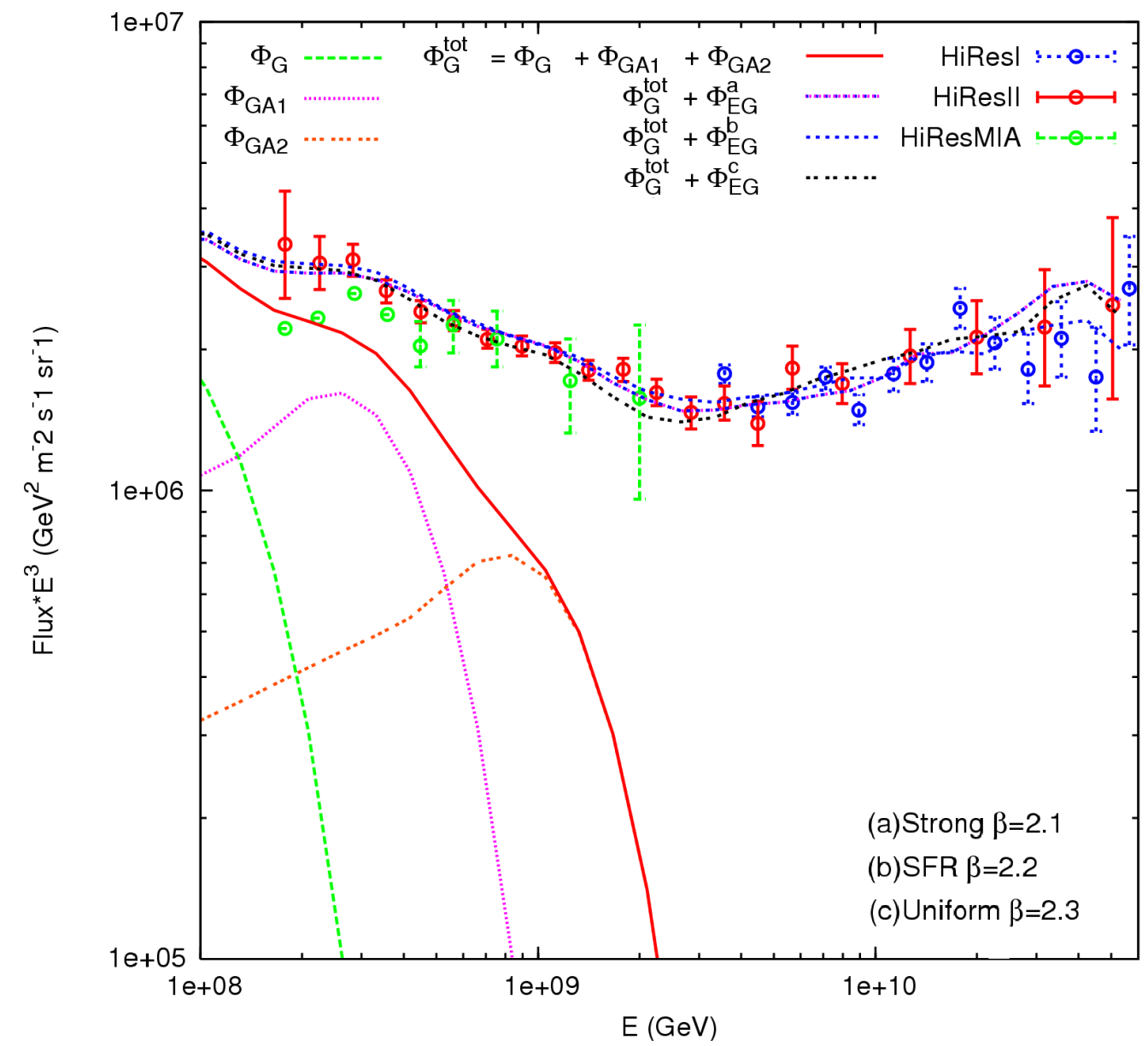

Fig. 18. Combined total spectrum $\Phi_{G}^{t o t}+\Phi_{E G}^{a, b, c}$ renormalized to HiRes spectrum data for different EG evolution models ( $(2.3)$. The Galactic spectrum $\Phi_{G}$, the Galactic additional components $\Phi_{G A 1}, \Phi_{G A 2}$ and the total Galactic spectrum $\Phi_{G}^{t o t}$ are also shown. The normalizations of the two Galactic additional component are the ones determined for the EG "SFR" model.

Since the HiRes spectrum extends to lower energies than those of Auger, in this case we renormalize both additional Galactic components to match the HiResII data in the energy range $10^{17.3}-10^{18.3} \mathrm{eV}$ (Fig.18). The EG spectra, on the other hand, are already normalized to HiRes data (92.3).

As in the Auger case, the position of the ankle measured by HiRes ( $6 \times$ $10^{18} \mathrm{eV}$ ) is not accurately reproduced with any of the combined spectra if a reasonable agreement at higher energies is required. Consequently, we select for subsequent analysis the EG "SFR" model, which is the one that provides the best visual fit to the experimental data.

We assume, as in $\$ 3.3 .1$, that the composition of the EG spectrum and of the two Galactic additional components is a binary mixture of proton and Iron nuclei and compute their relative abundances in order to match the $\left\langle X_{\max }\right\rangle$ energy dependence of the HiRes data. 

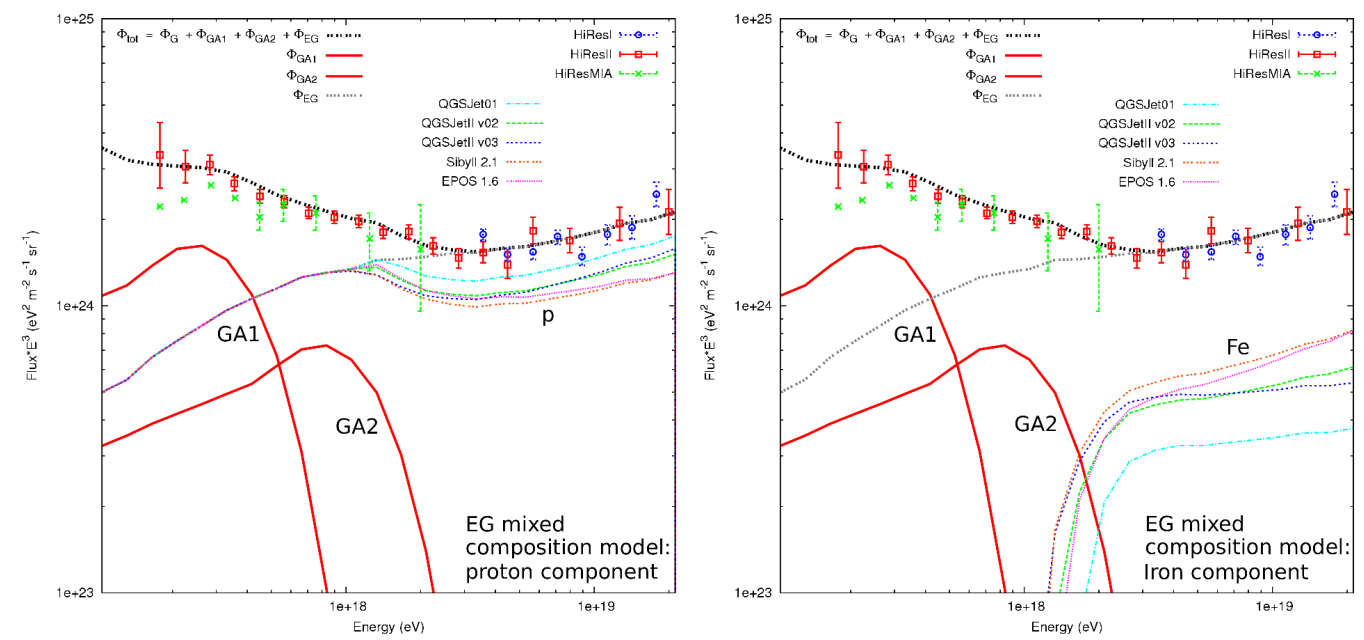

(a) EG composition: proton (left) and Iron (right) components.
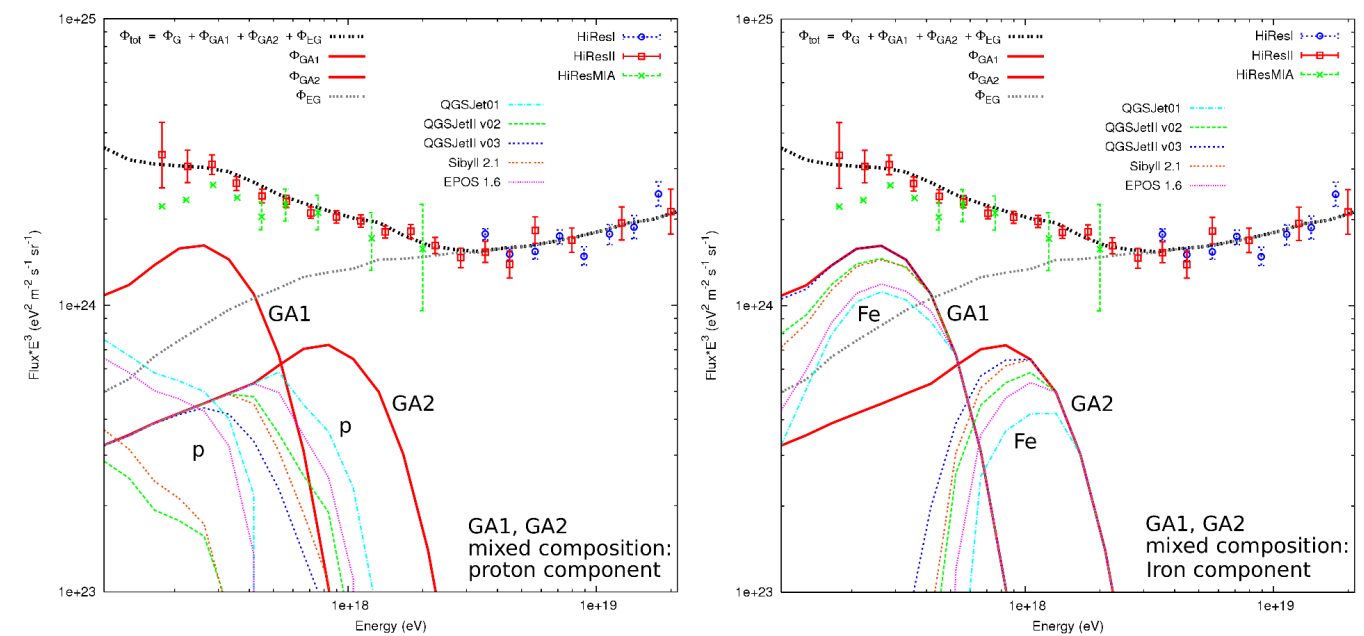

(b) GA1 and GA2 composition: proton (left) and Iron (right) components.

Fig. 19. HiRes data. (a) EG proton and Iron composition; (b) GA1 and GA2 proton and Iron compositions. The fluxes are calculated for each HIM in order to reproduce the HiRes and HiResMIA $\left\langle X_{\max }\right\rangle$ data. The Galactic additional components $\left(\Phi_{G A 1}, \Phi_{G A 2}\right)$, the EG spectrum $\left(\Phi_{E G}\right)$ and the total spectrum $\left(\Phi_{t o t}=\Phi_{G}+\Phi_{G A 1}+\Phi_{G A 2}+\Phi_{E G}\right)$ are also shown, as well as the HiRes spectrum data.

The compositions as a function of energy of EG, GA1 and GA2 are determined for all the HIM in the energy range of HiRes and HiResMIA $X_{\max }$ data $\sim 10^{17}$ to $\sim 2 \times 10^{19}$.

The results for the calculated Iron and proton fluxes for GA1, GA2 and EG are shown in Fig.19.

The average atomic weights, $\langle A\rangle$, are shown in Fig, 20, for each HIM. 

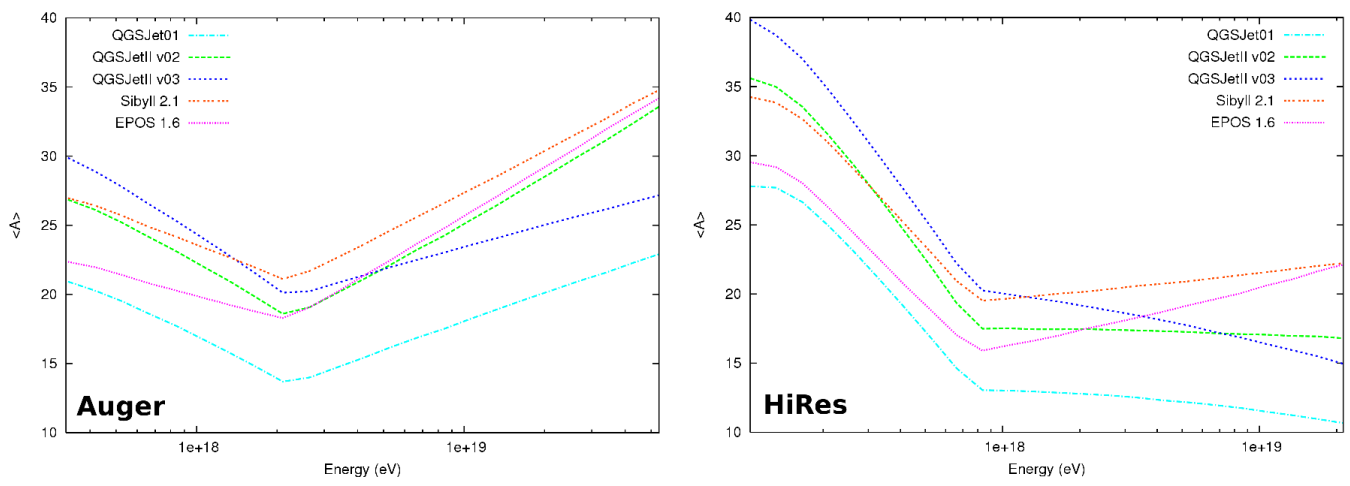

Fig. 20. Average atomic weight of the combined cosmic ray flux: the values were obtained from the calculated EG, GA1 and GA2 compositions in order to reproduce the Auger (Fig.17) and HiRes (Fig 19) $\left\langle X_{\max }\right\rangle$ data, under different HIM assumptions.

\subsection{Discussion on composition}

In section 3.2 we calculated the $X_{\max }$ dependence on energy for all the possible scenarios obtained by combining the calculated SNR Galactic spectrum with different extragalactic models (\$2.4). Several HIMs were used.

Simple assumptions were made on the composition of the two additional Galactic components. The first one, which is required by both EG models, was assumed to be Iron dominated, as it is probably contributed by compact and highly magnetized SNRs. The origin and properties of GA2, required in the case of a mixed composition EG spectrum, are more uncertain. Consequently, as an exploratory test, we considered in section 3.2 two cases for GA2: a pure proton and a pure Iron composition.

For all the possible combined spectra, we used different parameterizations of $\left\langle X_{\max }\right\rangle$ obtained for the HIMs used in literature to calculate the behavior of $X_{\max }$ in the energy range $\left[10^{17} \mathrm{eV}, 10^{20} \mathrm{eV}\right]$. We compared the calculated $X_{\max }$ energy profiles with the available experimental data from Fly's Eye, HiRes, HiRes-MIA and Auger.

The EG proton model is very much compatible with HiRes-MIA data at energies below $10^{18} \mathrm{eV}$ for any HIM. However, if the current interpretation of $X_{\text {max }}$ in terms of shower composition is correct, the later model is completely incompatible with higher energy data which points to a mixed composition.

In the case of the EG mixed-composition model, also two energy regimes are clearly distinguishable.

At energies above $\sim 3 \times 10^{18}$ the slope of the elongation rate seems to be consistent with that of the data up to, possibly, $\sim 2 \times 10^{19} \mathrm{eV}$. The effect of different 
HIMs is basically to change the normalization of the curves, changing at the same time the experimental data set with which the models are more compatible. For energies smaller than $\sim 3 \times 10^{18} \mathrm{eV}$, the calculated $\left\langle X_{\max }\right\rangle$ profiles are very dependent on the assumption made regarding the composition of GA2. A heavy GA2 can fit reasonably well the Fly's Eye data, while a light GA1 is more consistent with HiRes-MIA in all the energy interval and with Auger between $4-6 \times 10^{17} \mathrm{eV}$. Between $\sim 6 \times 10^{17}$ and $\sim 2 \times 10^{18} \mathrm{eV}$ Auger data is more or less in-between both solutions. These results are qualitatively independent of the HIM. Even if the assumption of a pure chemical composition for GA1 and GA2 is artificial, it is clear that the present discrepancies between the various experimental results are of astrophysical significance, since they have quite different implications with respect to the nature of most powerful Galactic accelerators. However, and surprisingly enough, the uncertainty associated with the several HIM currently in use in the literature does not pose a too large qualitative problem from the astrophysical point of view; that is, unless our present understanding of the hadronic interaction processes is not, somehow, fundamentally wrong.

In sections $\$ 3.3 .1$ and $\$ 3.3 .2$ we determined the composition, as a function of energy, of the extragalactic spectrum (EG) and the two additional Galactic components (GA1, GA2) that fits $X_{\max }$ data of Auger and HiRes, under the simplifying assumption of a binary mixture of $\mathrm{p}$ and Fe for the cosmic ray flux.

The main result is that, regardless of the experimental data set considered, the composition has to be mixed to some extent along all the spectrum. The assumed HIM plays a very important quantitative role in the inferred composition of any individual component.

An important point to note regarding GA1 and GA2 is that, even if they have mixed composition, the composition profile inside each one of them is similar in the sense that the individual fluxes are lighter at lower energies and then become heavier as the energy increases. This is a systematic effect that is quantitatively, but not qualitatively affected by the HIM used. Profiles such of those we obtained for GA1 and GA2 are similar to what can be expected from different populations of SNRs immersed in differing environments. If this is correct, then probably only SNRs are required in order to explain the main part of the Galactic flux up to energies $\lesssim 3 \times 10^{18} \mathrm{eV}$. In any case, GA2 is lighter than GA1, which could be an evidence for the existence of a minor contribution coming from a different source like, for example, inductors associated with compact objects.

The estimated average atomic weight (see, Fig. 20) varies widely depending on the assumed HIM. However, it can be seen that, for a given HIM, HiRes always implies heavier compositions on the Galactic side of the transition region than 
Auger. Despite this systematic difference, both data sets show the same trend of a decreasing value of $\langle A\rangle$ as a function of energy.

The opposite happens at higher energies on the extragalactic side of the flux, where Auger implies an increasing $\langle A\rangle$ as a function of energy regardless of the HIM, and HiRes predicts either a rather constant or a much more slowly changing composition. Also, for the latter data set, the uncertainties introduced by the HIM are qualitatively more important, since the high energy slope of the profile can change signs depending on the adopted interaction model.

It must be noted that, for both data sets, there seems to be a break in the average energy composition profile at an energy around $\sim 10^{18} \mathrm{eV}$ or slightly higher, which may further highlight the physical association between the ankle feature in the cosmic ray spectrum an the Galactic-extragalactic flux transition.

\section{Conclusions}

We have analyzed the matching conditions of the Galactic and extragalactic components of cosmic rays along the second knee and the ankle.

We have calculated the diffusive Galactic spectrum from regular SNRs using the numerical diffusive propagation code GALPROP. We used this first assessment of the Galactic cosmic ray flux to analyze the matching conditions with two alternative models for the extragalactic flux: a pure proton model [2] and a mixed composition model [3].

The first step was the matching of the observed energy spectrum by combining the Galactic and extragalactic fluxes. From this process, it becomes clear that additional Galactic components must be at play in reality. The minimum amount of additional components that allows us to satisfactorily reproduce the spectrum is either one or two. The pure proton model is the less expensive one with only one required additional component, GA1. The mixed composition model on the other hand requires, besides GA1, another components at still higher energies, GA2.

If no other information is used, both theoretical models are indistinguishable from the experimental point of view. Therefore, we also analyzed the effect of incorporating composition information, in the form of elongation rate data $\left(\left\langle X_{\max }\right\rangle\right)$. For this study, the shape of each one of the components is determined from the matching of the observed total energy spectrum. The elongation rate of the combined fluxes is then fitted to the HiRes and Auger 
data sets by changing appropriately the composition as a function of energy of each one of the components, Galactic and extragalactic. In order to make the analysis simpler, only a binary mixture of $\mathrm{p}$ and $\mathrm{Fe}$ is considered. The main result is that the additional Galactic components, GA1 and GA2, must have a mixed composition. Furthermore, inside each one of this components there is a progressive evolution of the composition from lighter to heavier as the energy increases. This is consistent with this components being originated in different populations of SNRs. Additionally, GA2 is globally lighter than GA1, which could indicate the possible existence of a minor contribution from another acceleration mechanisms, without a rigidity cut-off, operating at the highest energies.

The uncertainties introduced by the HIM are always important from the quantitative point of view. From the qualitative point of view, however, there are some results that seem rather independent of the HIM, like the diminution in average atomic mass along the low energy branch of the ankle and the existence of a discontinuity in the slope of the energy profile of $\langle A\rangle$ around the mid ankle. These results seem also to be supported by both, Auger and HiRes.

Our present results may be certainly considered as preliminary due to experimental uncertainties and simplifications in the numerical approach. First, there is the paucity of data involved in the determination of the energy spectrum in the region encompassing the second knee and the ankle and the divergence between $\left\langle X_{\max }\right\rangle$ measurements by different experiments below $3 \times 10^{18}$ $\mathrm{eV}$. A proper experimental characterization of this very important region will likely have to wait until the release of the KASCADE-Grande [77] and Auger enhancement data [72. Second, there are arguable simplifying assumptions related with our diffusive treatment of the Galactic component at the highest energies which is, very likely, undergoing a change in propagation to a full ballistic regime. This can be somehow mitigated by the fact that Fe nuclei should still be diffusive inside this energy interval, while protons would only deviate importantly from the diffusive approximation at the highest energies considered for the Galactic flux. In any case, the importance of the transition region as a play ground for disentangling the Galactic and extragalactic cosmic ray fluxes is unquestionable and considerable effort should be invested in its full experimental characterization and theoretical modeling.

\section{Acknowledgments}

CDD is partially supported by $\mathrm{PhD}$ grant from the Università degli Studi di Milano. CDD also thanks the Inst. de Ciencias Nucleares, UNAM, for its hospitality during several extended stays. GMT thanks CONACyT and PAPIIT/CIC-UNAM, through grants IN115707 and IN115607, for partial sup- 
port. Both authors have benefitted from their interaction with several colleagues from the Pierre Auger Collaboration.

\section{References}

[1] G. A. Medina-Tanco, Ultra-high energy cosmic rays: from GeV to ZeV, Proc. EMA 2005, astro-ph/0607543 (2006) 1-34.

[2] V. Berezinsky, A. Gazizov, S. Grigorieva, On astrophysical solution to ultrahigh energy cosmic rays, PhRvD 74 (4) (2006) 043005-+.

[3] D. Allard, A. V. Olinto, E. Parizot, Signatures of the extragalactic cosmicray source composition from spectrum and shower depth measurements, ArXiv Astrophysics e-prints, astro-ph/0703633.

[4] T. Antoni, et al. for the KASCADE collaboration, KASCADE measurements of energy spectra for elemental groups of cosmic rays: Results and open problems, Astroparticle Physics 24 (2005) 1-25.

[5] P. L. Biermann, G. A. Medina-Tanco, Ultra high energy cosmic ray sources and experimental results, Nuclear Physics B Proceedings Supplements 122 (2003) $86-97$.

[6] A. M. Hillas, Cosmic Rays: Recent Progress and some Current Questions, ArXiv Astrophysics e-prints, astro-ph/0607109.

[7] A. M. Hillas, TOPICAL REVIEW: Can diffusive shock acceleration in supernova remnants account for high-energy galactic cosmic rays?, Journal of Physics G Nuclear Physics 31 (2005) 95-+.

[8] A. M. Hillas, Where do $10^{19}$ eV cosmic rays come from?, Nuclear Physics B Proceedings Supplements 136 (2004) 139-146.

[9] T. Wibig, A. W. Wolfendale, At what particle energy do extragalactic cosmic rays start to predominate?, J. Phys. G31 (2005) 255-264.

[10] E. Waxman, Cosmological Gamma-Ray Bursts and the Highest Energy Cosmic Rays, Physical Review Letters 75 (1995) 386-389.

[11] M. Vietri, The Acceleration of Ultra-High-Energy Cosmic Rays in Gamma-Ray Bursts, ApJ 453 (1995) 883-+.

[12] C. T. Hill, D. N. Schramm, Ultrahigh-energy cosmic-ray spectrum, PhRvD 31 (1985) 564-580.

[13] V. Berezinsky, A. Z. Gazizov, S. I. Grigorieva, Signatures of AGN model for UHECR, ArXiv Astrophysics e-prints, astro-ph/0210095.

[14] M. Takeda, et al., Energy determination in the Akeno Giant Air Shower Array experiment, Astroparticle Physics 19 (2003) 447-462. 
[15] R. U. Abbasi, et al., Measurement of the Flux of Ultrahigh Energy Cosmic Rays from Monocular Observations by the High Resolution Fly's Eye Experiment, Physical Review Letters 92 (15) (2004) 151101-+.

[16] M. I. Pravdin, et al., Energy Spectrum of Primary Cosmic Rays in the Energy Region of $10^{17}-10^{20}$ eV by Yakutsk Array Data, in: International Cosmic Ray Conference, Vol. 1 of International Cosmic Ray Conference, 2003, pp. 389-+.

[17] V. P. Egorova, et al., The spectrum features of UHECRs below and surrounding GZK, Nuclear Physics B Proceedings Supplements 136 (2004) 3-11.

[18] D. J. Bird, et al., The Cosmic ray energy spectrum observed by the Fly's Eye, Astrophys. J. 424 (1994) 491-502.

[19] L. Perrone, for the the Pierre Auger Collaboration, Measurement of the UHECR energy spectrum from hybrid data of the Pierre Auger Observatory, Proc. of 30th Int. Cosmic Ray Conf. 2007 (Merida, Yucatan, Mexico) [astro-ph/0706.2643].

[20] V. Berezinsky, On origin of ultra high energy cosmic rays, Ap\&SS 309 (2007) $453-463$.

[21] R. Aloisio, et al., A dip in the UHECR spectrum and the transition from galactic to extragalactic cosmic rays, Astroparticle Physics 27 (2007) 76-91.

[22] V. S. Berezinsky, S. I. Grigorieva, B. I. Hnatyk, Extragalactic UHE proton spectrum and prediction for iron-nuclei flux at $10^{8}-10^{9} \mathrm{GeV}$, Astroparticle Physics 21 (2004) 617-625.

[23] M. Kachelrieß, D. V. Semikoz, Reconciling the ultra-high energy cosmic ray spectrum with Fermi shock acceleration, Physics Letters B 634 (2006) 143-147.

[24] D. Allard, et al., UHE nuclei propagation and the interpretation of the ankle in the cosmic-ray spectrum, A\&A 443 (2005) L29-L32.

[25] D. Allard, E. Parizot, A. V. Olinto, On the transition from Galactic to extragalactic cosmic-rays: spectral and composition features from two opposite scenarios, ArXiv Astrophysics e-prints, astro-ph/0512345.

[26] P. L. Biermann, et al., Cosmic Rays from PeV to ZeV, Stellar Evolution, Supernova Physics and Gamma Ray Bursts, ArXiv Astrophysics e-prints, astro$\mathrm{ph} / 0302201$.

[27] A. W. Strong, I. V. Moskalenko, Propagation of Cosmic-Ray Nucleons in the Galaxy, ApJ 509 (1998) 212.

[28] A. W. Strong, I. V. Moskalenko, Models for galactic cosmic-ray propagation, "http://galprop.stanford.edu/web_galprop/galprop_home.html", Adv. Sp. Res. 27 (2001) 717.

[29] W. H. Press, et al., Numerical recipes in FORTRAN. The art of scientific computing, Cambridge: University Press, —c1992, 2nd ed., 1992.

[30] J. A. Simpson, M. Garcia-Munoz, Cosmic-ray lifetime in the Galaxy Experimental results and models, Space Science Reviews 46 (1988) 205-224. 
[31] P. L. Biermann, Cosmic rays. 1. The cosmic ray spectrum between $10^{4} \mathrm{GeV}$ and $310^{9}$ GeV, A\&A 271 (1993) 649-+.

[32] G. Case, D. Bhattacharya, Revisiting the galactic supernova remnant distribution., A\&AS 120 (1996) C437+.

[33] A. W. Strong, et al., The distribution of cosmic-ray sources in the Galaxy, $\gamma$ rays and the gradient in the CO-to- $\mathrm{H}_{2}$ relation, A\&A 422 (2004) L47-L50.

[34] T. K. Gaisser, Cosmic Rays and Particle Physics, Cosmic Rays and Particle Physics, by Thomas K. Gaisser, pp. 295. ISBN 0521326 672. Cambridge, UK: Cambridge University Press, January 1991., 1991.

[35] R. J. Protheroe, R. W. Clay, Ultra High Energy Cosmic Rays, Publications of the Astronomical Society of Australia 21 (2004) 1-22.

[36] G. F. Krymskii, A regular mechanism for the acceleration of charged particles on the front of a shock wave, Soviet Physics Doklady 22 (1977) 327-+.

[37] R. D. Blandford, J. P. Ostriker, Particle acceleration by astrophysical shocks, Apjl 221 (1978) L29-L32.

[38] A. Hesse, et al., Isotopic composition of silicon and iron in the galactic cosmic radiation., A\&A 314 (1996) 785-794.

[39] M. A. Duvernois, et al., The Isotopic Composition of Galactic Cosmic-Ray Elements from Carbon to Silicon: The Combined Release and Radiation Effects Satellite Investigation, ApJ 466 (1996) 457.

[40] M. A. Duvernois, J. A. Simpson, M. R. Thayer, Interstellar propagation of cosmic rays: analysis of the ULYSSES primary and secondary elemental abundances., A\&A 316 (1996) 555-563.

[41] M. A. Duvernois, M. R. Thayer, The Elemental Composition of the Galactic Cosmic-Ray Source: ULYSSES High-Energy Telescope Results, ApJ 465 (1996) 982.

[42] M. R. Thayer, An Investigation into Sulfur Isotopes in the Galactic Cosmic Rays, ApJ 482 (1997) 792.

[43] J. J. Connell, J. A. Simpson, Isotopic Abundances of Fe and Ni in Galactic Cosmic-Ray Sources, ApJ Lett. 475 (1997) L61.

[44] I. V. Moskalenko, et al., Secondary Antiprotons and Propagation of Cosmic Rays in the Galaxy and Heliosphere, ApJ 565 (2002) 280.

[45] A. W. Strong, J. R. Mattox, Gradient model analysis of EGRET diffuse Galactic $\gamma$-ray emission., A\&A 308 (1996) L21-L24.

[46] M. A. Gordon, W. B. Burton, Carbon monoxide in the Galaxy. I - The radial distribution of CO, H2, and nucleons, ApJ 208 (1976) 346-353.

[47] J. M. Dickey, F. J. Lockman, H I in the Galaxy, ARA\&A 28 (1990) 215-261. 
[48] P. Cox, E. Kruegel, P. G. Mezger, Principal heating sources of dust in the galactic disk, A\&A 155 (1986) 380-396.

[49] J. M. Cordes, et al., The Galactic distribution of free electrons, Nature 354 (1991) 121-124.

[50] T. A. Porter, et al., A new estimate of the Galactic interstellar radiation field between 0.1um and 1000um, in: International Cosmic Ray Conference, Vol. 4 of International Cosmic Ray Conference, 2005, pp. 77-+.

[51] I. V. Moskalenko, T. A. Porter, A. W. Strong, Attenuation of Very High Energy Gamma Rays by the Milky Way Interstellar Radiation Field, ApJ Lett. 640 (2006) L155-L158.

[52] D. R. Bergman, Monocular UHECR Spectrum Measurements from HiRes, in: International Cosmic Ray Conference, Vol. 7 of International Cosmic Ray Conference, 2005, pp. 307-+.

[53] T. Abu-Zayyad, et al., Measurement of the Cosmic-Ray Energy Spectrum and Composition from $10^{17}$ to $10^{18.3}$ eV Using a Hybrid Technique, ApJ 557 (2001) 686-699.

[54] M. Nagano, et al., Energy spectrum of primary cosmic rays between $10^{14.5}$ and $10^{18} \mathrm{eV}$., Journal of Physics G Nuclear Physics 10 (1984) 1295-1310.

[55] M. Nagano, et al., Energy spectrum of primary cosmic rays above $10^{17.0} \mathrm{eV}$ determined from extensive air shower experiments at Akeno., Journal of Physics G Nuclear Physics 18 (1992) 423-442.

[56] M. A. Lawrence, R. J. O. Reid, A. A. Watson, The cosmic ray energy spectrum above $4 \times 10^{17} \mathrm{eV}$ as measured by the Haverah Park array., Journal of Physics G Nuclear Physics 17 (1991) 733-757.

[57] M. Ave, et al., The energy spectrum of cosmic rays in the range $3 \times 10^{17}-4 \times 10^{18}$ $\mathrm{eV}$ as measured with the Haverah Park array, Astroparticle Physics 19 (2003) $47-60$.

[58] M. Roth, for the Auger Collaboration, Measurement of the UHECR energy spectrum using data from the Surface Detector of the Pierre Auger Observatory, Proc. of 30th Int. Cosmic Ray Conf. 2007 (Merida, Yucatan, Mexico) [astro$\mathrm{ph} / 0706.2096]$.

[59] J. W. Fowler, et al., A measurement of the cosmic ray spectrum and composition at the knee, Astroparticle Physics 15 (2001) 49-64.

[60] M. Amenomori, et al., Measurement of air shower cores to study the cosmic ray composition in the knee energy region, PhRvD 62 (7) (2000) 072007-+.

[61] S. Ozawa, Tibet Asgamma Collaboration, The Energy Spectrum of All-Particle Cosmic Rays around the Knee Region Observed with the Tibet Air-Shower Array, in: International Cosmic Ray Conference, Vol. 1 of International Cosmic Ray Conference, 2003, pp. 143-+. 
[62] N. Ito, et al., in: International Cosmic Ray Conference, Vol. 4 of International Cosmic Ray Conference, 1997, pp. 117-+.

[63] I. P. Ivanenko, V. Y. Shestoperov, et al., Energy Spectra of Cosmic Rays above $2 \mathrm{TeV}$ as Measured by the 'SOKOL' Apparatus, in: International Cosmic Ray Conference, Vol. 2 of International Cosmic Ray Conference, 1993, pp. 17-+.

[64] L. M. Cherry, K. Asakimori, et al., Cosmic Ray Proton and Helium Spectra Combined JACEE Results, in: International Cosmic Ray Conference, Vol. 2 of International Cosmic Ray Conference, 1995, pp. 728-+.

[65] K. Asakimori, et al., Cosmic-Ray Proton and Helium Spectra: Results from the JACEE Experiment, ApJ 502 (1998) 278-+.

[66] T. Grigorov et al. after Shibata, Energy spectrum and primary composition from direct measurements., Nuclear Physics B Proceedings Supplements 75 (1999) $22-27$.

[67] N. L. Grigorov, et al., in: International Cosmic Ray Conference, Vol. 5 of International Cosmic Ray Conference, 1971, pp. 1746, 1752, 1760.

[68] V. A. Derbina, et al., Cosmic-Ray Spectra and Composition in the Energy Range of 10-1000 TeV per Particle Obtained by the RUNJOB Experiment, ApJ Lett. 628 (2005) L41-L44.

[69] V. Berezinsky, Transition from galactic to extragalactic cosmic rays, Invited talk at 30th Int. Cosmic Ray Conf., Merida (Mexico) 2007 additional item of discussion added, typos corrected, [astro-ph/0710.2750].

[70] T. Stanev, P. L. Biermann, T. K. Gaisser, Cosmic rays. IV. The spectrum and chemical composition above $10^{4}$ GeV, A\&A 274 (1993) 902-+.

[71] P. L. Biermann, G. A. Medina Tanco, R. Engel, G. Pugliese, The last Gamma Ray Burst in our Galaxy? On the observed cosmic ray excess at particle energy 1 EeV, Ap. J. Letters 604 (2004) L29-32.

[72] G. Medina-Tanco, for the Pierre Auger Collaboration, Astrophysics Motivation behind the Pierre Auger Southern Observatory Enhancements, Proc. of 30th Int. Cosmic Ray Conf. 2007 (Merida, Yucatan, Mexico) [astro-ph/0709.0772].

[73] A. D. Supanitsky, et al., Underground Muon Counters as a Tool for Composition Analyses, ArXiv Astrophysics e-prints, astro-ph/0804.1068, submitted to Astroparticle Physiscs.

[74] R. U. Abbasi, et al., A Study of the Composition of Ultra-High-Energy Cosmic Rays Using the High-Resolution Fly's Eye, ApJ 622 (2005) 910-926.

[75] D. J. Bird, et al., Evidence for correlated changes in the spectrum and composition of cosmic rays at extremely high energies, Physical Review Letters 71 (1993) 3401-3404. 
[76] M. Unger, for the Pierre Auger Collaboration, Study of the Cosmic Ray Composition above 0.4 EeV using the Longitudinal Profiles of Showers observed at the Pierre Auger Observatory, Proc. of 30th Int. Cosmic Ray Conf. 2007 (Merida, Yucatan, Mexico) [astro-ph/0706.1495].

[77] M. Bertaina, et al., KASCADE-Grande: An overview and first results, Nuclear Instruments and Methods in Physics Research A 588 (2008) 162-165. 